\title{
Tight Hamilton cycles in cherry-quasirandom 3-uniform hypergraphs
}

\author{
Elad Aigner-Horev* and Gil Levy \\ Department of Mathematics and Computer Science, Ariel University, Israel \\ *Corresponding author. Email: horev.elad@gmail.com
}

(Received 12 December 2017; revised 5 August 2020; first published online 12 October 2020)

\begin{abstract}
We employ the absorbing-path method in order to prove two results regarding the emergence of tight Hamilton cycles in the so-called two-path or cherry-quasirandom 3-graphs.

Our first result asserts that for any fixed real $\alpha>0$, cherry-quasirandom 3-graphs of sufficiently large order $n$ having minimum 2-degree at least $\alpha(n-2)$ have a tight Hamilton cycle.

Our second result concerns the minimum 1-degree sufficient for such 3-graphs to have a tight Hamilton cycle. Roughly speaking, we prove that for every $d, \alpha>0$ satisfying $d+\alpha>1$, any sufficiently large $n$-vertex such 3-graph $H$ of density $d$ and minimum 1-degree at least $\alpha\left(\begin{array}{c}n-1 \\ 2\end{array}\right)$ has a tight Hamilton cycle.
\end{abstract}

2020 MSC Codes: Primary: 05C35, 05C65; Secondary: 05C80, 05D05, 05C42

\section{Introduction}

A theorem of Dirac [11] asserts that an $n$-vertex $(n \geqslant 3)$ graph whose minimum degree is at least $n / 2$ contains a Hamilton cycle; moreover, the degree condition imposed here is best possible. A rich and extensive body of work now exists concerning the extent to which Dirac's result can be extended to uniform hypergraphs; see e.g. [4], [8], [9], [13], [14], [15], [16], [17], [19], [20], [22], [23], [33], [34], [35], [36] and [38]. ${ }^{\mathrm{a}}$ Allow us to not reproduce here the intricate development of these results, as outstanding accounts of these already exist in the excellent surveys [24], [32] and [44].

We confine ourselves to 3-uniform hypergraphs (3-graphs hereafter). A 3-graph $C$ is said to form a loose cycle if its vertices can be cyclically ordered such that each edge of $C$ captures three vertices appearing consecutively in the ordering, every vertex is contained in an edge, and any two consecutive $^{\mathrm{b}}$ edges meet in precisely one vertex. We say that $C$ forms a tight cycle if there exists a cyclic ordering of its vertices such that every three consecutive vertices in this ordering define an edge of $C$; in particular, this implies that any two consecutive edges meet in precisely two vertices.

For a 3-graph $H$ and two of its distinct vertices $u$ and $v$, define

$$
\begin{aligned}
\operatorname{deg}_{H}(v) & :=\left|N_{H}(v)\right|:=\left|\left\{\{x, y\} \in\left(\begin{array}{c}
V(H) \\
2
\end{array}\right):\{x, y, v\} \in E(H)\right\}\right|=|\{e \in E(H): v \in e\}|, \\
\operatorname{deg}_{H}(u, v) & :=\left|N_{H}(u, v)\right|:=|\{w \in V(H):\{u, v, w\} \in E(H)\}|=|\{e \in E(H):\{u, v\} \subset e\}| .
\end{aligned}
$$

\footnotetext{
${ }^{\text {a }}$ The study of perfect matchings in hypergraphs is intimately related to the Hamiltonicity problem. We omit references to such results as our work here was not directly influenced by this line of research.

${ }^{b}$ Order of the edges inherited from the ordering of the vertices. 
We refer to $\operatorname{deg}_{H}(v)$ as the degree of $v$ (alternatively, 1-degree) and to $\operatorname{deg}_{H}(u, v)$ as the codegree of $u$ and $v$ (alternatively, 2-degree). Set

$$
\delta(H):=\min _{v \in V(H)} \operatorname{deg}_{H}(v) \text { and } \delta_{2}(H):=\min _{\{u, v\} \in\left(\begin{array}{c}
V(H) \\
2
\end{array}\right)} \operatorname{deg}_{H}(u, v)
$$

Resolving a conjecture of [19], first approximately [35] and then accurately [38], the latter result asserts that a sufficiently large $n$-vertex 3-graph $H$ satisfying $\delta_{2}(H) \geqslant\lfloor n / 2\rfloor$ contains a tight Hamilton cycle. A construction appearing in [19] demonstrates that the codegree condition imposed here is best possible. However, finding the correct threshold for $\delta(H)$ at which a 3-graph $H$ admits a tight Hamilton cycle remained elusive for quite some time. The problem has come to be known as the 5/9-conjecture [32, Conjecture 2.18] asserting that sufficiently large $n$-vertex 3-graphs $H$ satisfying $\delta(H) \geqslant(5 / 9+o(1))\left(\begin{array}{c}n-1 \\ 2\end{array}\right)$ admit a tight Hamilton cycle. Constructions appearing in [32] and [33] establish that the degree condition appearing in this conjecture is (asymptotically) best possible. The authors of [7] established that such 3-graphs admit a tight cycle covering all but $o(n)$ of the vertices. Recently, in a major breakthrough [30] (preceded by the deep result of [34] and around the same time as [7]), the 5/9-conjecture has been resolved.

An additional result relevant to our account is that of [27]. Presentation of the latter requires a brief overview regarding quasirandom 3-graphs. Launched in [5], [41] and [42], the study of quasirandom graphs has developed into a rich and vast theory; see e.g. [21]. While a canonical definition of quasirandom graphs was already captured in [5], [41] and [42], for hypergraphs the pursuit after a definition extending [5] took much longer. An elaborate account regarding the development of this pursuit can be seen in [1], [6], [25], [26] and [43] and references therein. Only recently with the work of [43] has this pursuit came to an end; an alternative combinatorial approach to the functional analytic work of [43] appears in [1].

Roughly speaking, for $k \geqslant 3$ each set system of $[k]=\{1, \ldots, k\}$ forming a maximal anti-chain gives rise to a notion of quasirandomness for $k$-graphs. In the case of interest to us, i.e. $k=3$, each of the maximal anti-chains

$$
\{\{1\},\{2\},\{3\}\}, \quad\{\{1,2\},\{3\}\}, \quad\{\{1,2\},\{2,3\}\} \quad \text { and } \quad\{\{1,2\},\{2,3\},\{1,3\}\}
$$

defines a notion of quasirandomness referred to as $*$-quasirandomness with $* \in\{\bullet \therefore, \therefore$, $\therefore$, $\therefore$ - $\}$, respectively (concrete definitions follow below); here these notions are arranged from left to right in increasing order of strength, so to speak.

A solid understanding of $\therefore$-quasirandomness (i.e. the weakest notion) was attained in [6] and [25]. More generally, we now know from [1] and [43] (and owing much to [26]) that all these notions are well-separated and form a certain hierarchy with $\therefore$-quasirandomness at the 'bottom' as the weakest notion (so it forms the broadest class of hypergraphs). In what follows, however, we will not be bothered with these notions of quasirandomness per se. Instead we shall consider weaker related notions. Borrowing notation from [29] and [28], given $d, \rho \in(0,1]$, an $n$-vertex 3-graph $H$ is said to be $(\rho, d) \cdot \because$-dense if

$$
e_{H}(X, Y, Z):=|\{(x, y, z) \in X \times Y \times Z:\{x, y, z\} \in E(H)\}| \geqslant d|X||Y||Z|-\rho n^{3}
$$

holds for every $X, Y, Z \subseteq V(H)$. If $\rho$ and $d$ exist yet are not made explicit, then we say that $H$ is $\therefore$-dense. The notion of $\therefore$-quasirandomness comes about if one imposes on $e_{H}(X, Y, Z)$ the upper bound corresponding to (1.1).

Returning to Hamiltonicity, one encounters the following remarkable result of [27] stated here for 3-graphs only.

Theorem 1.1 ([27]). For every $d, \alpha \in(0,1]$ there exist an $n_{0}$ and a $\rho>0$ such that the following holds whenever $n \geqslant n_{0}$ and even. Let $H$ be an $n$-vertex $(\rho, d) \bullet-$-dense 3 -graph satisfying $\delta(H) \geqslant$ $\alpha\left(\begin{array}{c}n-1 \\ 2\end{array}\right)$. Then $H$ admits a loose Hamilton cycle. 
Theorem 1.1 settles the issue of emergence of loose Hamilton cycles in quasirandom 3-graphs for any notion of quasirandomness and any type of degree (the latter owing to [32, Remark 1.4]). It asserts that all minimum degree conditions sufficient for the emergence of loose Hamilton cycles in quasirandom 3-graphs are degenerate (i.e. any positive $\alpha$ suffices). ${ }^{\mathrm{C}}$

For tight cycles, however, a result analogous to Theorem 1.1 does not exist for $\bullet \bullet$-quasirandom 3-graphs. Indeed, [27, Proposition 4] asserts that for every $\rho>0$ and a sufficiently large $n$, an $n$-vertex $(\rho, 1 / 8) \bullet$-quasirandom 3 -graph $H$ exists satisfying $\delta(H) \geqslant(1 / 8-\rho)\left(\begin{array}{c}n-1 \\ 2\end{array}\right)$ and having no tight Hamilton cycle. The constant $1 / 8$ here is not best possible, though, as the following construction demonstrates. Let $n \in \mathbb{N}$ be sufficiently large and let $V=X \dot{\cup} Y$ be a set of $n$ vertices such that $|X|=2 n / 3+1$ and $|Y|=n / 3-1$ (assume $3 \mid n$ ). Let $G \sim G(n, p)$ be the random graph put on $V$ where each edge is put in $G$ independently at random with probability $p$; we determine $p$ below. Define $H$ to be the 3-graph whose set of vertices is $V$ and whose set of edges consists of:

- all the sets $e \in\left(\begin{array}{c}V \\ 3\end{array}\right)$ satisfying $G[e] \cong K_{3}$ and $e \subseteq X$ or $e \subseteq Y$ or $|e \cap X|=1$,

- together with the sets $e \in\left(\begin{array}{c}V \\ 3\end{array}\right)$ satisfying $2=|e \cap X|:=|\{u, v\}|$ and $u v \notin E(G)$.

An argument similar to the one used in [33, Construction 2] asserts that $H$ has no tight Hamilton cycle. Indeed, no tight path can connect a triple contained in $X$ with a vertex of $Y$. Consequently, if $H$ were to admit a tight Hamilton cycle $C$ then $X$ must be an independent set in $C$ and $Y$ a vertexcover of $C$. With this together with the fact that $C$ is 3-regular (i.e. with respect to 1-degree), we reach $n=e(C) \leqslant \sum_{y \in Y} \operatorname{deg}_{C}(y)=3|Y|<n$, a contradiction. Every triple $e$ is taken into $H$ either with probability $p^{3}$ or $1-p$. We set $p^{3}=1-p$, so that $p=0.6823$. Using binomial tail estimations, it follows that it is highly likely that $H$ would have edge density $\approx 0.3177$, satisfy $\delta(H) \approx 0.245 n^{2}$ and be $\because$-dense. We acknowledge the discussions [31] regarding this construction.

Replacing the degree condition seen in Theorem 1.1 with a codegree condition would be insufficient to yield a result analogous to Theorem 1.1. Indeed, in [27] it is indicated that an adaption of the construction seen in [27, Proposition 4] yields a $\bullet \bullet$-dense graph $H$ with $\delta_{2}(H) \geqslant n / 9$ admitting no tight Hamilton cycle.

\subsection{Our results}

If we were to 'climb' up the hierarchy of notions of quasirandomness for 3-graphs and strengthen the quasirandomness condition satisfied by the host 3-graph, would we then encounter an analogue of Theorem 1.1 for tight Hamilton cycles? Let $d, \rho \in(0,1]$. An $n$-vertex 3 -graph $H$ is called $(\rho, d) \therefore$-dense if

$$
e_{H}\left(\vec{G}_{1}, \vec{G}_{2}\right):=\left|\left\{(x, y, z) \in \mathcal{P}_{2}\left(\vec{G}_{1}, \vec{G}_{2}\right):\{x, y, z\} \in E(H)\right\}\right| \geqslant d\left|\mathcal{P}_{2}\left(\vec{G}_{1}, \vec{G}_{2}\right)\right|-\rho n^{3}
$$

holds for every $\vec{G}_{1}, \vec{G}_{2} \subseteq V(H) \times V(H)$, where

$$
\mathcal{P}_{2}\left(\vec{G}_{1}, \vec{G}_{2}\right):=\left\{(x, y, z) \in V(H)^{3}:(x, y) \in \vec{G}_{1},(y, z) \in \vec{G}_{2}\right\}
$$

If $\rho$ and $d$ exist yet are not made explicit, then we say that $H$ is $\therefore$-dense (pronounced cherrydense).

Our first main result asserts that the minimum codegree condition sufficient to imply the emergence of a tight Hamilton cycle in $\therefore$-dense 3-graphs is degenerate.

Theorem 1.2. For every $d, \alpha \in(0,1]$, there exist an integer $n_{0}$ and a real $\rho>0$ such that the following holds for all $n \geqslant n_{0}$. Let $H$ be an $n$-vertex $(\rho, d)$-dense 3 -graph satisfying $\delta_{2}(H) \geqslant \alpha(n-2)$. Then $H$ has a tight Hamilton cycle.

${ }^{c}$ For hypergraphs with higher uniformity, the full version of Theorem 1.1 handles the emergence of the so-called 1-cycles. 
In Theorem 1.2, the parameter $d$ plays a somewhat docile role in the sense that no strict conditions other than it being fixed and positive need be imposed. For our second result, we consider $\therefore$-dense 3-graphs with an imposed minimum 1-degree condition. Here a condition on $d$ arises (for us) as follows.

Theorem 1.3. For every $d, \alpha \in(0,1]$ satisfying $\alpha+d>1$, there exist an integer $n_{0}$ and a real $\rho>0$ such that the following holds for all $n \geqslant n_{0}$. Let $H$ be an $n$-vertex $(\rho, d) .$. -dense 3-graph satisfying $\delta(H) \geqslant \alpha\left(\begin{array}{c}n-1 \\ 2\end{array}\right)$. Then $H$ has a tight Hamilton cycle.

Unlike Theorem 1.2, the requirement $\alpha+d>1$ does not allow for a degenerate minimum 1 -degree condition. Nevertheless, it is more flexible than other results mentioned thus far. We conjecture (with some hesitation) that the condition $\alpha+d>1$ appearing in Theorem 1.2 can be replaced with degenerate conditions for both $\alpha$ and $d$ as follows.

Conjecture 1.4. For every $d, \alpha \in(0,1]$ there exist an $n_{0}$ and $\rho>0$ such that the following holds for all $n \geqslant n_{0}$. Let $H$ be an $n$-vertex $(\rho, d) \therefore$-dense 3-graph satisfying $\delta(H) \geqslant \alpha\left(\begin{array}{c}n-1 \\ 2\end{array}\right)$. Then $H$ has a tight Hamilton cycle.

'Between' $\therefore$-quasirandomness and $\therefore$-quasirandomness, there lies $\because$-quasirandomness. For $d, \rho \in(0,1)$ an $n$-vertex 3 -graph $H$ is called $(\rho, d) \cdot-$-dense if

$$
e_{H}(\vec{P}, X):=|\{((u, v), x) \in \vec{P} \times X:\{u, v, x\} \in E(H)\}| \geqslant d|\vec{P}||X|-\rho n^{3}
$$

holds for every $\vec{P} \subseteq V(H) \times V(H)$ and every $X \subseteq V(H)$. Unlike $\therefore$-quasirandom 3-graphs, for which the Turán density of $K_{4}^{(3)}$ (the complete 3-graph on four vertices) is zero [29], the Turán density of $K_{4}^{(3)-}$ (i.e. $K_{4}^{(3)}$ with a single edge removed) in $\because$-quasirandom 3 -graphs is $1 / 4$ [28]. The absorbing configurations (see Section 4 for details) used in this account involve copies of $K_{4}^{(3)-}$. Consequently results in the spirit of Theorems 1.2 and 1.3 cannot possibly be attained for $\because$ - -quasirandom 3 -graphs using the absorbing-path method and the absorbing configurations used in our account. We subscribe to the point of view that the flaw is not in the method and that for $\because$-quasirandom 3 -graphs the minimum 1-degree and 2-degree conditions sufficient to imply tight Hamiltonicity are both non-degenerate. The fact that the Turán density of $K_{4}^{(3)-}$ in $\therefore$-quasirandom 3 -graphs coincides with that seen in $\therefore$-quasirandom 3 -graphs [28] makes it not far-fetched to suspect that the minimum degree conditions in $\{\bullet \bullet, \bullet$ - $\}$-quasirandom 3 -graphs coincide as well.

Open problems. Are the following true?

- For every $d>1 / 3$ and $\varepsilon>0$, there exist an integer $n_{0}$ and a real $\rho>0$ such that the following holds whenever $n \geqslant n_{0}$. Let $H$ be an $n$-vertex $(\rho, d)$ :-dense 3 -graph satisfying $\delta_{2}(H) \geqslant$ $n / 3+\varepsilon n$. Then $H$ has a tight Hamiltonian cycle.

- For every $d>1 / 2$ and $\varepsilon>0$, there exist an integer $n_{0}$ and a real $\rho>0$ such that the following holds whenever $n \geqslant n_{0}$. Let $H$ be an $n$-vertex $(\rho, d)$ :-dense 3-graph satisfying $\delta_{1}(H) \geqslant$ $n^{2} / 4+\varepsilon n$. Then $H$ has a tight Hamilton cycle.

- In the two questions above replace $\bullet$-denseness with $\bullet \bullet$-denseness.

During the review and revision of this manuscript, Araújo, Piga and Schacht [2] announced that they have proved that for every $\varepsilon>0$ there exists a $\rho>0$ such that every sufficiently large $(\rho, 1 / 4+\varepsilon) \bullet$-dense 3 -graph $H$ satisfying $\delta(H) \geqslant \varepsilon\left(\begin{array}{c}n-1 \\ 2\end{array}\right)$ contains a tight Hamilton cycle, and, 
moreover, that the constant $1 / 4$ is optimal. Their result implies our Theorem 1.3 and settles some of the questions appearing above. At the time of writing these lines the full proof of their result was not available to us.

\subsection{Our approach}

We employ the so-called absorbing-path method introduced in [35] and further developed in [36] and [37]. Roughly speaking, this method reduces the problem of finding a tight Hamilton cycle to that of finding a tight cycle supporting two properties. First, it covers all but $\zeta n$ vertices for some carefully chosen fixed 'small' $\zeta \in(0,1)$. Second, it contains a special path referred to as an absorbing-path (rigorously defined below), which has the capability of being rerouted using only those 'missing' $\zeta n$ vertices while keeping its ends unchanged, and in this manner absorb, so to speak, all missing vertices rendering a tight Hamilton cycle. Numerous reincarnations of this method now exist in the literature; see e.g. [14], [27], [30] and [34]. We consequently omit a more rigorous outline of this method and proceed directly to the statement of the so-called pillar lemmas underlying this method; these are the so-called connecting lemma, absorbing-path lemma, path-cover lemma and reservoir lemma.

By a $k$-path we mean a 3 -graph $P$ on $k$ vertices and $k-2$ edges such that there exists a labelling of $V(P)$, namely $v_{1}, \ldots, v_{k}$, such that $\left\{v_{i}, v_{i+1}, v_{i+2}\right\} \in E(P)$ for every $i \in[1, k-2]$. It is said that $P$ connects the pairs $\left\{v_{1}, v_{2}\right\}$ and $\left\{v_{k-1}, v_{k-2}\right\}$, also referred to as the end-pairs or simply the ends of $P$. Throughout, the term path is used to mean a tight path.

Roughly speaking, in the absorbing-path method, the role of the connecting lemma is, as its name suggests, to connect two disjoint pairs of vertices via a short path. A trivial precondition for such a lemma is that the given pairs that are to be connected both admit some non-trivial codegree. The 3-graphs of Theorem 1.2 come equipped with a minimum codegree assumption which, although degenerate, will be sufficient to establish such a lemma owing to the $\therefore$-denseness of the host 3-graph. The 3-graphs of Theorem 1.3, however, do not support a minimum codegree condition. As a result we will require two separate connecting lemmas, one for each of our main results.

Our connecting lemma suitable for Theorem 1.2 reads as follows.

Lemma 1.1 (connecting lemma: 2-degree). For every $d_{1.1}, \alpha_{1.1} \in(0,1]$, there exist an integer $n_{1.1}:=n_{1.1}\left(d_{1.1}, \alpha_{1.1}\right)$ and a real $\rho_{1.1}:=\rho_{1.1}\left(d_{1.1}, \alpha_{1.1}\right)>0$ such that the following holds for all $n \geqslant n_{1.1}$ and $0<\rho<\rho_{1.1}$.

Let $H$ be an $n$-vertex $\left(\rho, d_{1.1}\right)$-dense 3-graph satisfying $\delta_{2}(H) \geqslant \alpha_{1.1}(n-2)$ and let $\{x, y\}$ and $\left\{x^{\prime}, y^{\prime}\right\}$ be two disjoint pairs of vertices. Then there exists a 10-path in $H$ connecting $\{x, y\}$ and $\left\{x^{\prime}, y^{\prime}\right\}$.

The premise of Theorem 1.3 allows for 3-graphs with pairs of vertices having codegree zero or one that is too modest for our methods to work. Fortunately, regardless of any degree conditions, $\therefore$-dense 3 -graphs admit a certain statistical minimum codegree condition in the sense that most pairs of vertices admit a meaningful codegree. This we make precise below in (2.3). Unlike Lemma 1.1, then, the connecting lemma suitable for Theorem 1.3 appeals to this statistical minimum codegree condition, and upon a judicious choice of parameters connects pairs of vertices whose codegree is sufficiently high. It is in this lemma that we encounter the following function $g(\cdot)$. Given reals $x, y>0$ satisfying $x+y>1$, let

$$
g(x, y):=\min \{x, y,(x+y-1) /(y+1)\} .
$$

The inequality $\alpha+d>1$ appearing in Theorem 1.3 traces back to the third term of this function. 
Lemma 1.2 (connecting lemma: 1-degree). For every $d_{1.2}, \alpha_{1.2}, \eta_{1.2} \in(0,1]$, satisfying $\alpha_{1.2}+$ $d_{1.2}>1$, and $\eta_{1.2}<g\left(\alpha_{1.2}, d_{1.2}\right)$, there exist an integer $n_{1.2}:=n_{1.2}\left(d_{1.2}, \alpha_{1.2}, \eta_{1.2}\right)$ and a real $\rho_{1.2}:=$ $\rho_{1.2}\left(d_{1.2}, \alpha_{1.2}, \eta_{1.2}\right)>0$ such that the following holds for all $n \geqslant n_{1.2}$ and $0<\rho<\rho_{1.2}$.

Let $H$ be an $n$-vertex $\left(\rho, d_{1.2}\right) \ldots$-dense 3-graph satisfying $\delta(H) \geqslant \alpha_{1.2}\left(\begin{array}{c}n-1 \\ 2\end{array}\right)$ and let $\{x, y\}$ and $\left\{x^{\prime}, y^{\prime}\right\}$ be two disjoint pairs of vertices each having codegree at least $\left(d_{1.2}-\eta_{1.2}\right) n$. Then there exists a 10-path in H connecting $\{x, y\}$ and $\left\{x^{\prime}, y^{\prime}\right\}$.

It is in fact true that for $\therefore$-dense 3 -graphs, a connecting lemma imposing no minimum degree conditions of any kind is possible. Such a lemma is presented in Lemma 3.2 in Section 3.3. Alas, for our needs this lemma is insufficient and this too is explained in Section 3.3.

A path $A$ in an $n$-vertex 3-graph $H$, is said to be $m$-absorbing if, for every set $U \subseteq V(H) \backslash V(A)$ with $|U| \leqslant m$, there is a path $A_{U}$ having the same ends as $A$ and satisfying $V\left(A_{U}\right)=V(A) \cup U$. A path is said to be a $(\beta, \mu, \kappa)$-absorbing-path if it is $\mu n$-absorbing, has length at most $\kappa n$ and both its ends have codegree at least $\beta n$ in $H$.

The 'split' between the aforementioned connecting lemmas propagates (for us) onwards onto the absorbing-path lemmas, leading to a need to support two separate such lemmas. The absorbing-path lemma suitable for Theorem 1.2 reads as follows.

Lemma 1.3 (absorbing-path lemma: 2-degree). For every $d_{1.3}, \alpha_{1.3}, \beta_{1.3} \in(0,1]$ such that $\beta_{1.3}<\min \left\{d_{1.3}, \alpha_{1.3}\right\}$, there exist an integer $n_{1.3}:=n_{1.3}\left(d_{1.3}, \alpha_{1.3}, \beta_{1.3}\right)$, a real $\rho_{1.3}:=$ $\rho_{1.3}\left(d_{1.3}, \alpha_{1.3}, \beta_{1.3}\right)>0$, a real $0<\kappa_{1.3}:=\kappa_{1.3}\left(d_{1.3}, \alpha_{1.3}, \beta_{1.3}\right) \leqslant \beta_{1.3} / 2$ and a real $\mu_{1.3}:=$ $\mu_{1.3}\left(d_{1.3}, \alpha_{1.3}\right)>0$ such that the following holds whenever $n \geqslant n_{1.3}$ and $0<\rho<\rho_{1.3}$.

If $H$ is an $n$-vertex $\left(\rho, d_{1.3}\right) .$. -dense 3-graph satisfying $\delta_{2}(H) \geqslant \alpha_{1.3}(n-2)$, then it admits a $\left(\beta_{1.3}, \mu_{1.3}, \kappa_{1.3}\right)$-absorbing-path.

The condition $\beta_{1.3}<\min \left\{d_{1.3}, \alpha_{1.3}\right\}$ appearing in the last lemma seems somewhat puzzling in view that part of the premise of the lemma is that $\delta_{2}(H) \geqslant \alpha_{1.3}(n-2)$. To a certain extent, this condition can be mitigated. We incur it here due to having certain ingredients required for the proofs of Lemma 1.3 and its counterpart consolidated, namely Lemma 1.4 stated next. This then mandates that $\beta_{1.3}<d_{1.3}$ be imposed to render subsequent applications of (2.3) meaningful. The condition $\beta_{1.3}<\alpha_{1.3}$ is admittedly 'artificial'; it is kept for brevity, as seen in the proof of Lemma 1.3.

Given two reals $\alpha, \beta>0$, we write $\beta \ll \alpha$ to indicate that these can be set such that $\beta$, while fixed, can be chosen arbitrarily smaller than $\alpha$. The aforementioned counterpart of Lemma 1.3 suitable for the setting of Theorem 1.3 reads as follows.

Lemma 1.4 (absorbing-path lemma: 1-degree). For every $d_{1.4}, \alpha_{1.4}, \eta_{1.4} \in(0,1]$ satisfying $\alpha_{1.4}+d_{1.4}>1$ and $\eta_{1.4}<g\left(\alpha_{1.4}, d_{1.4}\right)$, there exist an integer $n_{1.4}:=n_{1.4}\left(d_{1.4}, \alpha_{1.4}, \eta_{1.4}\right)$, a real $\rho_{1.4}:=\rho_{1.4}\left(d_{1.4}, \alpha_{1.4}, \eta_{1.4}\right)>0$, a real $0<\kappa_{1.4}:=\kappa_{1.4}\left(d_{1.4}, \alpha_{1.4}, \eta_{1.4}\right) \ll \eta_{1.4}$ and a real $\mu_{1.4}:=$ $\mu_{1.4}\left(d_{1.4}, \alpha_{1.4}, \eta_{1.4}\right)>0$ such that the following holds whenever $n \geqslant n_{1.4}$ and $0<\rho<\rho_{1.4}$.

If $H$ is an $n$-vertex $\left(\rho, d_{1.4}\right)$-dense 3-graph satisfying $\delta(H) \geqslant \alpha_{1.4}\left(\begin{array}{c}n-1 \\ 2\end{array}\right)$, then it admits a $\left(d_{1.4}-\eta_{1.4}, \mu_{1.4}, \kappa_{1.4}\right)$-absorbing-path.

For the next pillar lemma, $\therefore$-denseness is not required. Here a weaker notion of denseness suffices. Let $d, \rho \in(0,1]$ and let $H$ be an $n$-vertex 3-graph. If

$$
e_{H}(X):=\left|E(H) \cap\left(\begin{array}{c}
X \\
3
\end{array}\right)\right| \geqslant d\left(\begin{array}{c}
|X| \\
3
\end{array}\right)-\rho n^{3}
$$


holds for every $X \subseteq V(H)$, then $H$ is said to be $(\rho, d)$-dense. If $\rho$ and $d$ are known to exist yet are not made explicit, then we say that $H$ is 1 -set-dense. ${ }^{\mathrm{d}}$ The following lemma imposes no minimum degree conditions on the 3-graph. It will be used in the proofs of both Theorem 1.2 and Theorem 1.3.

Lemma 1.5 (path-cover lemma). For every $d_{1.5}, \zeta_{1.5} \in(0,1]$, there exist $n_{1.5}:=n_{1.5}\left(d_{1.5}, \zeta_{1.5}\right)$, $\rho_{1.5}=\rho_{1.5}\left(d_{1.5}, \zeta_{1.5}\right)>0$ and an integer $\ell_{1.5}=\ell_{1.5}\left(d_{1.5}, \zeta_{1.5}\right)$ such that the following holds for all $n \geqslant n_{1.5}$ and $0<\rho<\rho_{1.5}$.

Let $H$ be an $n$-vertex $\left(\rho, d_{1.5}\right)$-dense 3-graph. Then all but at most $\zeta_{1.5} n$ vertices of $H$ can be covered using at most $\ell_{1.5}$ vertex-disjoint paths.

The fourth and last pillar lemma is the reservoir lemma. We employ the all-encompassing ${ }^{\mathrm{e}}$ reservoir lemma of [34], which will service both Theorem 1.2 and Theorem 1.3.

Lemma 1.6 (reservoir lemma [34, Lemma 3.10]). Let $U_{1}, \ldots, U_{s}$ be subsets of an $n$-element set $V$ and let $L_{1}, \ldots, L_{k}$ be graphs on $V$, where $s:=s(n)$ and $k:=k(n)$ are both polynomials in $n$ and such that for sequences of constants $\left(\alpha_{i} \in(0,1)\right)_{i \in[s]}$ and $\left(\beta_{j} \in(0,1)\right)_{j \in[k]}$, we have $\left|U_{i}\right| \geqslant \alpha_{i} n$ for every $i \in[s]$ and $e\left(L_{j}\right) \geqslant \beta_{j}\left(\begin{array}{c}n \\ 2\end{array}\right)$ for every $j \in[k]$.

Then, for every constant $v_{1.6} \in(0,1)$, there exists an $n_{1.6}:=n_{1.6}\left(v_{1.6}\right)$ such that if $n \geqslant n_{1.6}$, then there exists a subset $R \subseteq V$ satisfying

(R.1) ||$R\left|-v_{1.6} n\right| \leqslant v_{1.6} n^{2 / 3}$,

(R.2) for all $i \in[s],\left|U_{i} \cap R\right| \geqslant\left(\alpha_{i}-2 n^{-1 / 3}\right)|R|$ holds, and

(R.3) for all $j \in[k], e\left(L_{j}[R]\right) \geqslant\left(\beta_{j}-3 n^{-1 / 3}\right)\left(\begin{array}{c}|R| \\ 2\end{array}\right)$ holds.

Lemma 1.6 is something of an overkill as far as Theorem 1.2 is concerned; indeed, the proof of the latter relies rather weakly only on (R.1) and (R.2). The proof of Theorem 1.3, though, requires the full force of Lemma 1.6, so to speak. In particular, it crucially relies on the $n^{-1 / 3}$-terms seen in Lemma 1.6. A reservoir lemma akin to what is seen in [35] indeed suffices for Theorem 1.2. The latter, however, is subsumed by Lemma 1.6 and thus omitted.

Constants. For most of this account, we tend to keep track of the raw values of the involved constants. We do, however, appeal on occasion to the notation $\ll$ defined above for constants.

\section{Pairs with positive codegree}

Let $H$ be a 3-graph, let $\vec{G} \subseteq V(H) \times V(H)$ and let $(u, v) \in V(H) \times V(H)$. We write

$$
\operatorname{deg}_{H}(u, v, \vec{G}):=\mid\{z \in V(H):((z, u) \in \vec{G} \text { or }(u, z) \in \vec{G}) \text { and }\{z, u, v\} \in E(H)\} \mid
$$

to denote the number of edges $\{z, u, v\} \in E(H)$ for which at least one of the (ordered) pairs $(z, u)$ or $(u, z)$ is present in the directed graph $\vec{G}$. Note that $\operatorname{deg}_{H}(u, v, \vec{G}) \neq \operatorname{deg}_{H}(v, u, \vec{G})$ is possible. In this definition, $\vec{G}$, although directed, is treated as an undirected graph and indeed below we shall also write $\operatorname{deg}_{H}(u, v, G)$ when $G$ is an undirected graph.

We find it more convenient to have the following lemma formulated using undirected graphs.

\footnotetext{
${ }^{\mathrm{d}}$ If, in addition to (1.4), $H$ also satisfies its corresponding upper bound, then $H$ is $\bullet \bullet$-quasirandom (see e.g. [6]).

eAll-encompassing in the sense that it can handle the 1-degree and 2-degree settings in one stroke.
} 
Lemma 2.1. Let $d, \alpha$ and $\rho$ be positive reals and let $H$ be $a(\rho, d) \therefore$-dense n-vertex 3-graph. Let $G$ be a graph on $V(H)$ and let $Y \subseteq V(G)$ satisfy

$$
\operatorname{deg}_{G}(y) \geqslant k \text { for all } y \in Y
$$

where $k:=k(n)$ is an integer. For an integer $\Delta:=\Delta(n)$, set

$$
\vec{B}_{\Delta}:=\left\{(y, z) \in Y \times V(H): \operatorname{deg}_{H}(y, z, G)<\Delta\right\} .
$$

Then

$$
\left|\vec{B}_{\Delta}\right| \leqslant \frac{\rho n^{3}}{d k-\Delta}
$$

Proof. Let $G_{Y} \subseteq G$ be the subgraph of $G$ induced by the edges incident to $Y$. Define ${ }^{f}$

$$
\vec{G}_{Y}:=\{(v, y):\{v, y\} \in E(G), v \in V(H), y \in Y\} \subseteq V(H) \times Y .
$$

Then

$$
d \cdot\left|\mathcal{P}_{2}\left(\vec{G}_{Y}, \vec{B}_{\Delta}\right)\right|-\rho n^{3} \leqslant e_{H}\left(\vec{G}_{Y}, \vec{B}_{\Delta}\right)<\left|\vec{B}_{\Delta}\right| \cdot \Delta
$$

Recalling that

$$
\mathcal{P}_{2}\left(\vec{G}_{Y}, \vec{B}_{\Delta}\right)=\left\{(x, y, z): x, z \in V(H), y \in Y,(x, y) \in \vec{G}_{Y},(y, z) \in \vec{B}_{\Delta}\right\}
$$

we may write

$$
\left|\mathcal{P}_{2}\left(\vec{G}_{Y}, \vec{B}_{\Delta}\right)\right| \geqslant \sum_{y \in Y} \operatorname{deg}_{G_{Y}}(y)\left|\left\{(y, z):(y, z) \in \vec{B}_{\Delta}\right\}\right| \stackrel{(2.1)}{\geqslant} k \sum_{y \in Y}\left|\left\{(y, z):(y, z) \in \vec{B}_{\Delta}\right\}\right|=k\left|\vec{B}_{\Delta}\right| .
$$

Then we obtain

$$
d k\left|\vec{B}_{\Delta}\right|-\rho n^{3}<\left|\vec{B}_{\Delta}\right| \cdot \Delta
$$

The claim now follows upon isolating $\left|\vec{B}_{\Delta}\right|$ in the last inequality.

For an $n$-vertex $(\rho, d) \ldots$-dense 3 -graph $H$ and a fixed real $\beta>0$, define

$$
B_{\beta}:=B_{\beta}(H):=\left\{\{u, v\} \in\left(\begin{array}{c}
V(H) \\
2
\end{array}\right): \operatorname{deg}_{H}(u, v)<\beta n\right\}
$$

to consist of all unordered pairs of vertices whose codegree is smaller than $\beta n$. An argument akin to setting $G$ to be the complete graph on $V(H), Y=V(H), k=n-1$ and $\Delta=\beta n$ in Lemma 2.1 yields an upper bound on $\left|B_{\beta}\right|$. To see this, consider

$$
d\left|\mathcal{P}_{2}\left(V(H) \times V(H), \vec{B}_{\beta}\right)\right|-\rho n^{3} \leqslant e_{H}\left(V(H) \times V(H), \vec{B}_{\beta}\right) \leqslant\left|\vec{B}_{\beta}\right| \cdot \beta n,
$$

where $\vec{B}_{\beta}:=\left\{(u, v),(v, u):\{u, v\} \in B_{\beta}\right\}$. Then

$$
d n\left|\vec{B}_{\beta}\right|-\rho n^{3} \leqslant\left|\vec{B}_{\beta}\right| \cdot \beta n,
$$

so that upon isolating $\vec{B}_{\beta}$ we arrive at

$$
2\left|B_{\beta}\right|=\left|\vec{B}_{\beta}\right| \leqslant \frac{\rho n^{3}}{d n-\beta n}=\frac{\rho}{d-\beta} n^{2} .
$$

In particular, ignoring the factor of $1 / 2$, we may write

$$
\left|B_{\beta}\right| \leqslant \frac{\rho}{d-\beta} n^{2}
$$

the latter makes sense only if $\beta<d$.

\footnotetext{
${ }^{\mathrm{f}}$ Here an edge $y y^{\prime} \in E(G)$ with $y, y^{\prime} \in Y$ gives rise to two pairs in $\vec{G}_{Y}$.
} 
A consequence of (2.3) is that the set of edges

$$
E_{<\beta}:=E_{<\beta}(H):=\left\{e \in E(H): \exists\{u, v\} \in\left(\begin{array}{l}
e \\
2
\end{array}\right) \text { satisfying } \operatorname{deg}_{H}(u, v)<\beta n\right\}
$$

satisfies

$$
\left|E_{<\beta}\right| \leqslant\left|B_{\beta}\right| \cdot n \leqslant \frac{\rho}{d-\beta} n^{3} .
$$

The spanning subgraph $H_{\beta} \subseteq H$ induced by $E(H) \backslash E_{<\beta}$ then consists only of edges each pair of which has codegree at least $\beta n$ in $H$. On its own, $H_{\beta}$ may admit no meaningful minimum degree condition. It does, however, satisfy

$$
e_{H_{\beta}}\left(\vec{G}_{1}, \vec{G}_{2}\right) \geqslant e_{H}\left(\vec{G}_{1}, \vec{G}_{2}\right)-\left|E_{<\beta}\right| \geqslant d\left|\mathcal{P}_{2}\left(\vec{G}_{1}, \vec{G}_{2}\right)\right|-\rho\left(1+\frac{1}{d-\beta}\right) n^{3}
$$

for all $\vec{G}_{1}, \vec{G}_{2} \subseteq V(H) \times V(H)=V\left(H_{\beta}\right) \times V\left(H_{\beta}\right)$. Consequently, upon a judicious choice of constants, $H_{\beta}$ inherits (in the sense of (2.4)) a certain level of $\therefore$-denseness from $H$. This feature arises in the proof of Theorem 1.3 seen in Section 6.2.

\section{Connecting lemmas}

In this section we prove Lemmas 1.1 and 1.2. In terms of graphs, our approach for both of these lemmas can be crudely described as follows. In order to connect two prescribed vertices, a sequence of neighbourhoods, called a cascade, is cultivated, one from each vertex. This continues until these neighbourhoods expand sufficiently so as to render a certain quasirandomness assumption non-trivial, giving rise to numerous 'links' between the two sequences of neighbourhoods. Two paths are then traced backwards from a 'link' to the two prescribed vertices via the two sequences of neighbourhoods, all the while maintaining vertex-disjointness of the paths thus traced.

\subsection{Connecting lemma: 2-degree setting}

In this section we prove Lemma 1.1, which is the connecting lemma suitable for Theorem 1.2. At the centre of our proof of Lemma 1.1 is the structure of cascades; the next section is dedicated to their definition.

\subsubsection{Cascades}

Let $n$ be a sufficiently large integer and let $H$ be an $n$-vertex 3-graph satisfying $\delta_{2}(H) \geqslant \beta n$ for some fixed real $\beta \in(0,1]$ independent of $n$ (and such that $\beta n \leqslant n-2$, naturally). Fix $x$ and $y$ to be two vertices in $H$. Below we define the tuple

$$
\mathcal{C}_{\beta}(x, y):=\left(x, y, N_{1}(x, y), N_{2}(x, y), N_{3}(x, y), G_{1}(x, y), G_{2}(x, y), G_{3}(x, y)\right)
$$

and refer to it as an $\{x, y\}_{\beta}$-cascade, with cascades being a term borrowed from [35]. All members of the above tuple depend on $\beta$ as well, but we omit this from the notation. In what follows, each of these members is defined. In broad terms, for every $i \in[3], N_{i}(x, y)$ denotes a set of vertices that essentially corresponds to the ith co-neighbourhood of the pair $\{x, y\}$. The parameters $\left(G_{i}(x, y)\right)_{i \in[3]}$ represent certain graphs between these co-neighbourhoods which will facilitate the tracking of 5-paths from $N_{3}(x, y)$ all the way (back) to $\{x, y\}$.

Let $N_{1}:=N_{1}(x, y):=N_{H}(x, y)$. The assumption $\delta_{2}(H) \geqslant \beta n$ implies that

$$
\left|N_{1}\right| \geqslant \beta n \text {. }
$$


Define $G_{1}:=G_{1}(x, y)$ to be the (bipartite) graph whose vertex set is $\{y\} \cup N_{1}$ and whose edges are given by the set $\left\{y z: z \in N_{1}\right\}$. To define $N_{2}:=N_{2}(x, y)$ and $G_{2}:=G_{2}(x, y)$, we proceed in two steps. For the first step, set

$$
N_{2}^{\prime}:=N_{2}^{\prime}(x, y):=\bigcup_{z \in N_{1}} N_{H}(y, z)=\left\{w \in V(H): \exists z \in N_{1} \text { such that }\{y, z, w\} \in E(H)\right\} .
$$

Define $G_{2}^{\prime}:=G_{2}^{\prime}(x, y)$ to be the graph whose vertex set is $N_{1} \cup N_{2}^{\prime}$ and whose edges are given by the set

$$
E\left(G_{2}^{\prime}\right):=\left\{z z^{\prime}: z \in N_{1}, z^{\prime} \in N_{2}^{\prime} \cap N_{H}(y, z)\right\}=\left\{z z^{\prime}: z \in N_{1}, z^{\prime} \in V(H) \text { and }\left\{y, z, z^{\prime}\right\} \in E(H)\right\} .
$$

The assumption that $\delta_{2}(H) \geqslant \beta n$ implies that $\operatorname{deg}_{G_{2}^{\prime}}(z) \geqslant \beta n$ for every $z \in N_{1}$. Then

$$
e\left(G_{2}^{\prime}\right) \geqslant \frac{1}{2} \sum_{z \in N_{1}} \operatorname{deg}_{G_{2}^{\prime}}(z) \geqslant\left|N_{1}\right| \beta n / 2 \stackrel{(3.1)}{\geqslant} \beta^{2} n^{2} / 2 .
$$

For the second step towards the definitions of $N_{2}:=N_{2}(x, y)$ and $G_{2}:=G_{2}(x, y)$, we discard members of $N_{2}^{\prime}$ whose degree in $G_{2}^{\prime}$ into $N_{1}$ is 'too low', as follows. Set

$$
N_{2}^{\text {(low) }}:=N_{2}^{\text {(low) }}(x, y):=\left\{z \in N_{2}^{\prime}: \operatorname{deg}_{G_{2}^{\prime}}(z)<\log n\right\} .
$$

(The choice of $\log n$ here is completely arbitrary. Any function $\omega(n) \ll n$ growing slowly to $\infty$ will suffice; this will become clear soon.) Setting $N_{2}:=N_{2}(x, y):=N_{2}^{\prime} \backslash N_{2}^{(\text {low })}$, we arrive at

$$
\beta^{2} n^{2} / 2 \stackrel{(3.3)}{\leqslant} e\left(G_{2}^{\prime}\right) \leqslant(\log n) \cdot\left|N_{2}^{(\text {low })}\right|+\left|N_{2}\right| \cdot\left|N_{1}\right| \leqslant n \log n+\left|N_{2}\right| \cdot n
$$

so that, for sufficiently large $n$,

$$
\left|N_{2}\right| \geqslant \beta^{2} n / 4
$$

Set $G_{2}:=G_{2}(x, y):=G_{2}^{\prime}\left[N_{1} \cup N_{2}\right]$. This concludes the definitions of $N_{2}:=N_{2}(x, y)$ and $G_{2}:=$ $G_{2}(x, y)$.

We turn to the definition of the set $N_{3}:=N_{3}(x, y)$ and the graph $G_{3}:=G_{3}(x, y)$. To that end, associate an auxiliary graph $\mathcal{B}_{w}:=\mathcal{B}_{w}(x, y)$ with every vertex $w \in N_{2}$. In particular, for a fixed vertex $w \in N_{2}$, let $\mathcal{B}_{w}$ be the graph whose vertex set is $V(H)$ and whose edges are given by the set

$$
E\left(\mathcal{B}_{w}\right):=\left\{u z: u \in V(H), z \in N_{G_{2}}(w) \subseteq N_{1} \text { and }\{z, w, u\} \in E(H)\right\} .
$$

Define

$$
N_{3}:=N_{3}(x, y):=\left\{u \in V(H): \exists w \in N_{2} \text { such that } \operatorname{deg}_{\mathcal{B}_{w}}(u) \geqslant 20\right\}
$$

and let $G_{3}:=G_{3}(x, y)$ be the graph whose vertex set is $N_{2} \cup N_{3}$ and whose edge set is given by

$$
E\left(G_{3}\right):=\left\{u w: u \in N_{3}, w \in N_{2} \text { and } \operatorname{deg}_{\mathcal{B}_{w}}(u) \geqslant 20\right\} .
$$

This completes the definition of an $\{x, y\}_{\beta}$-cascade.

We conclude this section by recording a few useful traits of $\{x, y\}_{\beta}$-cascades that will be called upon in subsequent arguments. Continuing with the notation set thus far, fix $w \in N_{2}$. Then

$$
\operatorname{deg}_{G_{2}}(w) \cdot \beta n \stackrel{\delta_{2}(H) \geqslant \beta n}{\leqslant} e\left(\mathcal{B}_{w}\right) \leqslant 20 \cdot s+(n-s) \operatorname{deg}_{G_{2}}(w)
$$

where $s$ denotes the number of vertices $u \in V(H)$ satisfying $\operatorname{deg}_{\mathcal{B}_{w}}(u)<20$. Then, for sufficiently large $n$, we obtain

$$
n-s \geqslant \beta n-\frac{20 \cdot s}{\operatorname{deg}_{G_{2}}(w)} \geqslant \beta n-\frac{20 \cdot n}{\log n} \geqslant \beta n / 2 .
$$


As $\operatorname{deg}_{G_{3}}(w)=n-s$, it follows that

$$
\operatorname{deg}_{G_{3}}(w) \geqslant \beta n / 2 \quad \text { for every } w \in N_{2} .
$$

This, in particular, implies that

$$
\left|N_{3}\right| \geqslant \beta n / 2 \text { and } e\left(G_{3}\right) \geqslant \frac{1}{2} \sum_{w \in N_{2}} \operatorname{deg}_{G_{3}}(w) \geqslant\left|N_{2}\right| \beta n / 4 \stackrel{(3.4)}{\geqslant} \beta^{2} n^{2} / 16 .
$$

\subsubsection{Links}

In addition to $\{x, y\}$ and $\mathcal{C}_{\beta}(x, y)$ defined in Section 3.1.1, let $\left\{x^{\prime}, y^{\prime}\right\}$ be a pair of vertices disjoint from $\{x, y\}$ and let $\mathcal{C}_{\beta}\left(x^{\prime}, y^{\prime}\right)$ be an $\left\{x^{\prime}, y^{\prime}\right\}_{\beta}$-cascade in $H$. A quadruple $(z, u, v, w) \in N_{2}(x, y) \times$ $N_{3}(x, y) \times N_{3}\left(x^{\prime}, y^{\prime}\right) \times N_{2}\left(x^{\prime}, y^{\prime}\right)$ is said to be an $\left(\{x, y\},\left\{x^{\prime}, y^{\prime}\right\}\right)$-link with respect to $\mathcal{C}_{\beta}(x, y)$ and $\mathcal{C}_{\beta}\left(x^{\prime}, y^{\prime}\right)$ if

(L.1) $x, y, z, u, v, w, y^{\prime}, x^{\prime}$ are all distinct,

(L.2) $\{z, u, v\},\{u, v, w\} \in E(H)$, and

(L.3) $z u \in E\left(G_{3}(x, y)\right)$ and $v w \in E\left(G_{3}\left(x^{\prime}, y^{\prime}\right)\right)$.

Lemma 3.1. If two distinct pairs of vertices, namely $\{x, y\}$ and $\left\{x^{\prime}, y^{\prime}\right\}$, admit an $\left(\{x, y\},\left\{x^{\prime}, y^{\prime}\right\}\right)$-link, then $H$ admits a 10-path connecting $\{x, y\}$ and $\left\{x^{\prime}, y^{\prime}\right\}$.

Proof. Let

$$
(z, u, v, w) \in N_{2}(x, y) \times N_{3}(x, y) \times N_{3}\left(x^{\prime}, y^{\prime}\right) \times N_{2}\left(x^{\prime}, y^{\prime}\right)
$$

be an $\left(\{x, y\},\left\{x^{\prime}, y^{\prime}\right\}\right)$-link. First we construct a 5-path connecting $\{x, y\}$ and $\{z, u\}$ through $\mathcal{C}(x, y)$. Having $z u \in E\left(G_{3}(x, y)\right)$ means that $\operatorname{deg}_{\mathcal{B}_{z}}(u) \geqslant 20$, which in other words means that there are at least 20 vertices $z^{\prime} \in N_{G_{2}(x, y)}(z) \subseteq N_{1}(x, y)$ such that $\left\{z^{\prime}, z, u\right\} \in E(H)$. We may then choose one such vertex $z^{\prime}$ such that $z^{\prime} \in N_{1}(x, y) \backslash\left\{x, y, x^{\prime}, y^{\prime}, z, u, v, w\right\}$. Having $z z^{\prime} \in E\left(G_{2}(x, y)\right)$ implies that $\left\{y, z^{\prime}, z\right\} \in E(H)$. The 5-path is made complete with the fact that $\left\{x, y, z^{\prime}\right\} \in E(H)$. Let $P$ denote this path.

It remains to construct a 5-path through the cascade of $\left\{x^{\prime}, y^{\prime}\right\}$ connecting $\{v, w\}$ and $\left\{x^{\prime}, y^{\prime}\right\}$ in such a way as to not meet any vertex of $P$. The same argument used for constructing $P$ can be used here as well except for one change. This time around, we require a vertex $z^{\prime \prime} \in N_{2}\left(x^{\prime}, y^{\prime}\right)$ to play the corresponding role assumed by $z^{\prime}$ above. The vertex $z^{\prime \prime}$ must satisfy $z^{\prime \prime} \notin\left\{x, y, z^{\prime}, x^{\prime}, y^{\prime}, z, u, v, w\right\}$ (i.e. it has to avoid $z^{\prime}$ as well). Clearly there is enough freedom to do so.

\subsubsection{Proof of Lemma 1.1}

Given $d:=d_{1.1}, \alpha:=\alpha_{1.1}$ as in the premise of the lemma, set

$$
\rho_{1.1}(d, \alpha):=\frac{d \alpha^{6}}{2^{14}} .
$$

Let $0<\rho<\rho_{1.1}(d, \beta)$ be fixed, let $H$ be a $(\rho, d) \therefore$-dense 3-graph satisfying $\delta_{2}(H) \geqslant \alpha n$ (we naturally assume that $\alpha$ is such that $\alpha n \leqslant n-2)$, and let $\{x, y\}$ and $\left\{x^{\prime}, y^{\prime}\right\}$ be two disjoint pairs of vertices in $V(H)$.

By Lemma 3.1 it suffices to show that the cascades $\mathcal{C}_{\alpha}(x, y)$ and $\mathcal{C}_{\alpha}\left(x^{\prime}, y^{\prime}\right)$ taken in $H$ admit an $\left(\{x, y\},\left\{x^{\prime}, y^{\prime}\right\}\right)$-link (in $\left.H\right)$. Owing to (3.6), $e\left(G_{3}(x, y)\right) \geqslant \alpha^{2} n^{2} / 16$. There exists a subgraph $F \subseteq G_{3}(x, y)$ satisfying $\delta(F) \geqslant \alpha^{2} n / 16$ (see e.g. [10, Proposition 1.2.2]). Then

$$
\left|V(F) \cap N_{3}(x, y)\right| \geqslant \alpha^{2} n / 16
$$


Indeed, all edges in $G_{3}(x, y)$ (and thus in $F$ ) are of the form $N_{2}(x, y) \times N_{3}(x, y)$ (though $N_{2}(x, y) \cap$ $N_{3}(x, y)$ need not be empty). Hence there is a vertex $w \in N_{2}(x, y) \cap V(F)$. By definition $N_{F}(w) \subseteq$ $N_{G_{3}(x, y)}(w) \subseteq N_{3}(x, y)$ and (3.8) follows.

Set

$$
\vec{B}:=\left\{(z, u) \in\left(V(F) \cap N_{3}(x, y)\right) \times V(H): z \neq u \text { and } \operatorname{deg}_{H}(z, u, F)<d \alpha^{2} n / 32\right\} .
$$

By Lemma 2.1 applied with $G=F, Y:=V(F) \cap N_{3}(x, y), k=\alpha^{2} n / 16$ and $\Delta:=d \alpha^{2} n / 32$, we attain

$$
|\vec{B}| \leqslant \frac{\rho n^{3}}{d \alpha^{2} n / 16-d \alpha^{2} n / 32}=\frac{32 \rho}{d \alpha^{2}} \cdot n^{2} .
$$

A symmetrical argument applied to $\mathcal{C}_{\alpha}\left(x^{\prime}, y^{\prime}\right)$ asserts that the set

$$
\vec{B}^{\prime}:=\left\{(z, u) \in\left(V\left(F^{\prime}\right) \cap N_{3}\left(x^{\prime}, y^{\prime}\right)\right) \times V(H): z \neq u \text { and } \operatorname{deg}_{H}\left(z, u, F^{\prime}\right)<d \alpha^{2} n / 16\right\}
$$

satisfies $\left|\vec{B}^{\prime}\right| \leqslant 32 \rho d^{-1} \alpha^{-2} n^{2}$ as well, where $F^{\prime} \subseteq G_{3}\left(x^{\prime}, y^{\prime}\right)$ is the counterpart of $F$ in this argument (i.e. it is a subgraph of $G_{3}\left(x^{\prime}, y^{\prime}\right)$ satisfying $\left.\delta\left(F^{\prime}\right) \geqslant \alpha^{2} n / 16\right)$.

The set $\left(V(F) \cap N_{3}(x, y)\right) \times\left(V\left(F^{\prime}\right) \cap N_{3}\left(x^{\prime}, y^{\prime}\right)\right)$ has size at least $\alpha^{4} n^{2} / 2^{7}$, by (3.8); removing degenerate members (i.e. members of the form $(x, x)$ ), we retain at least $\alpha^{4} n^{2} / 2^{8}$ non-degenerate members of that Cartesian product. The latter set of non-degenerate pairs we denote by $\vec{T}$. Then

$$
\left|\vec{T} \backslash\left(\vec{B} \cup \vec{B}^{\prime}\right)\right| \geqslant|\vec{T}|-|\vec{B}|-\left|\vec{B}^{\prime}\right| \geqslant \frac{\alpha^{4}}{2^{8}} n^{2}-\frac{64 \rho}{d \alpha^{2}} n^{2} \stackrel{(3.7)}{\geqslant} \frac{\alpha^{4}}{2^{9}} n^{2} .
$$

Each member $(u, v) \in \vec{T} \backslash\left(\vec{B} \cup \vec{B}^{\prime}\right)$ satisfies $u \neq v, u \in V(F) \cap N_{3}(x, y), v \in V\left(F^{\prime}\right) \cap N_{3}\left(x^{\prime}, y^{\prime}\right)$, $\operatorname{deg}_{G}(u, v, F) \geqslant d \alpha^{2} n / 32$ and $\operatorname{deg}_{G}\left(v, u, F^{\prime}\right) \geqslant d \alpha^{2} n / 32$. That is, there are at least $d \alpha^{2} n / 32$ edges $\{u, v, z\} \in E(H)$ with $u z \in E(F)$ (so that $z \in N_{2}(x, y)$ ) and at least $d \alpha^{2} n / 32$ edges $\{u, v, w\} \in E(H)$ with $v w \in E\left(F^{\prime}\right)$ (so that $w \in N_{2}\left(x^{\prime}, y^{\prime}\right)$ ). Hence, for sufficiently large $n$, we may insist on (many choices) $w \neq z$ and thus form the required $\left\{(x, y),\left(x^{\prime}, y^{\prime}\right)\right\}$-link.

This completes the proof of Lemma 1.1 .

\subsection{Connecting lemma: 1-degree setting}

In this section we prove Lemma 1.2, which is the connecting lemma suitable for Theorem 1.3. The definition of cascades, seen in Section 3.1.1, fits any 3-graph $H$ satisfying $\delta_{2}(H)=\Omega(n)$. As such, the construction of cascades makes no appeal to $\therefore$-denseness. In Lemma 1.2, which is furnished with a minimum 1-degree condition only, cascades, as defined, are not at our disposal (at least not verbatim). To prove Lemma 1.2, then, we put forth a definition of a structure to which we refer as refined cascades. The latter is an adaption of cascades to the setting of Lemma 1.2.

Although we closely follow the definition of cascades when defining their refined counterparts, these two structures are quite different from one another. One crucial manifestation of this difference can be seen from the condition $\alpha+d>1$ stated in the premise of Theorem 1.3. This condition is, in fact, incurred through the definition of refined cascades. The construction of latter is then the sole 'bottleneck' in our approach preventing us from establishing Conjecture 1.4.

Unlike the case of cascades, the construction of their refined counterparts makes no appeal to $\therefore$-denseness of the host 3-graph. Consequently, the definitions of cascades and refined cascades are not consolidated.

\subsubsection{Refined cascades}

Let there be given $\alpha, d$ and $\eta$ satisfying $\alpha+d>1$ and

$$
0<\eta<g(\alpha, d)=\min \{\alpha, d,(\alpha+d-1) /(1+d)\} .
$$


The function $g(\cdot)$ is the one defined in (1.3). An appeal to the inequality $\alpha+d>1$ is made in the numerator of the third term appearing on the right-hand side of (3.9). Set an auxiliary constant

$$
0<\zeta:=\zeta(\alpha, d, \eta)<\eta+\eta d-\eta^{2},
$$

and set

$$
0<\rho<\min \{\eta / 4, \eta \zeta(d-\eta) / 4\}
$$

Let $H$ be a $(\rho, d) \therefore$-dense 3-graph satisfying $\delta(H) \geqslant \alpha\left(\begin{array}{c}n-1 \\ 2\end{array}\right)$. Setting $\beta=d-\eta$ in (2.3) yields

$$
\left|B_{d-\eta}\right| \leqslant \frac{\rho}{d-(d-\eta)} n^{2}=\frac{\rho}{\eta} n^{2} .
$$

Let $\{x, y\} \in\left(\begin{array}{c}V(H) \\ 2\end{array}\right)$ satisfying $\operatorname{deg}_{H}(x, y) \geqslant(d-\eta) n$ be fixed; by (3.12) coupled with the condition $\rho<\eta / 4$ stipulated in (3.11), there are $\Omega\left(n^{2}\right)$ such pairs in $H$.

In what follows, we define the tuple

$$
\mathcal{R}_{\alpha, d, \eta}(x, y):=\left(x, y, \mathcal{N}_{1}(x, y), \mathcal{N}_{2}(x, y), \mathcal{N}_{3}(x, y), \mathcal{G}_{1}(x, y), \mathcal{G}_{2}(x, y), \mathcal{G}_{3}(x, y)\right)
$$

and refer to it as an $\{x, y\}_{\alpha, d, \eta}$-refined-cascade. All members of this tuple depend on $\alpha, d$ and $\eta$ as well, but we omit this from the notation. The members $\mathcal{N}_{i}$ and $\mathcal{G}_{i}, i \in[3]$, assume roles analogous to those assumed by $N_{i}$ and $G_{i}, i \in$ [3], in the definition of cascades in Section 3.1.1. We proceed with the definition of each of the members of the above tuple.

Define

$$
\mathcal{N}_{1}:=\mathcal{N}_{1}(x, y):=N_{H}(x, y) \cap\left\{z \in V(H): \operatorname{deg}_{H}(z, y) \geqslant \eta n\right\} .
$$

Treating $B_{\eta}$ (see (2.2) for a definition) as a graph, we write

$$
\bar{B}_{\eta}:=\left\{\{u, v\} \in\left(\begin{array}{c}
V(H) \\
2
\end{array}\right): \operatorname{deg}_{H}(u, v) \geqslant \eta n\right\}
$$

to denote the graph complementing $B_{\eta}$ over $V(H)$. The set $\left\{z \in V(H): \operatorname{deg}_{H}(z, y) \geqslant \eta n\right\}$, appearing in (3.13), is then the neighbourhood of $y$ in $\bar{B}_{\eta}$. One is now reminded of the following remarkable fact established in [34, Claim 3.1], which in our setting (and owing to $\eta<\alpha$ as imposed in (3.9)) reads as follows.

Claim 1. [34, Claim 3.1] We have

$$
\delta\left(\bar{B}_{\eta}\right) \geqslant \frac{\alpha-\eta}{1-\eta}(n-1)
$$

By definition of $\{x, y\},\left|N_{H}(x, y)\right| \geqslant(d-\eta) n$; this, together with Claim 1, implies that if

$$
\frac{\alpha-\eta}{1-\eta}+d-\eta>1
$$

then $\left|\mathcal{N}_{1}\right|=\Omega(n)$. Rewriting this inequality as

$$
\alpha+d-1>\eta+\eta d-\eta^{2},
$$

we note that the inequality $\eta<(a+d-1) /(1+d)$ imposed in (3.9) implies that (3.14) is satisfied. Moreover, it is here at (3.14) that the condition $\alpha+d>1$, imposed in Theorem 1.3, stands out. It follows that

$$
\left|\mathcal{N}_{1}\right| \stackrel{(3.10)}{\geqslant} \zeta n
$$

Define $\mathcal{G}_{1}:=\mathcal{G}_{1}(x, y)$ to be the (bipartite) graph whose vertex set is given by $\{y\} \cup \mathcal{N}_{1}$ and whose edge set is given by $\left\{y z: z \in \mathcal{N}_{1}\right\}$. 
We proceed to defining $\mathcal{N}_{2}(x, y)$ and $\mathcal{G}_{2}(x, y)$. Set

$$
\mathcal{N}_{2}^{\prime}:=\mathcal{N}_{2}^{\prime}(x, y):=\bigcup_{z \in \mathcal{N}_{1}} N_{H}(y, z)=\left\{w \in V(H): \exists z \in \mathcal{N}_{1} \text { such that }\{y, z, w\} \in E(H)\right\},
$$

and define $\mathcal{G}_{2}^{\prime}:=\mathcal{G}_{2}^{\prime}(x, y)$ to be the graph whose vertex set is $\mathcal{N}_{1} \cup \mathcal{N}_{2}^{\prime}$ and whose edge set is given by

$$
E\left(\mathcal{G}_{2}^{\prime}\right):=\left\{z z^{\prime}: z \in \mathcal{N}_{1}, z^{\prime} \in V(H),\left\{y, z, z^{\prime}\right\} \in E(H)\right\} .
$$

By definition of $\mathcal{N}_{1}$ (see (3.13)), $\operatorname{deg}_{H}(y, z) \geqslant \eta n$ for every $z \in \mathcal{N}_{1}$ so that $\operatorname{deg}_{\mathcal{G}_{2}^{\prime}}(z) \geqslant \eta n$ holds for every $z \in \mathcal{N}_{1}$. Then

$$
e\left(\mathcal{G}_{2}^{\prime}\right) \geqslant \frac{1}{2} \sum_{z \in \mathcal{N}_{1}} \operatorname{deg}_{\mathcal{G}_{2}^{\prime}}(z) \geqslant\left|\mathcal{N}_{1}\right| \eta n / 2 \stackrel{(3.15)}{\geqslant} \zeta \eta n^{2} / 2 .
$$

All but at most $(\rho /(d-\eta)) n^{2}$ of the edges of $\mathcal{G}_{2}^{\prime}$ lie in $B_{\eta}$, by (2.3) (with $\beta=\eta$ in that equation). Consequently, there exists a subgraph $\mathcal{G}_{2}^{\prime \prime} \subseteq \mathcal{G}_{2}^{\prime}$ satisfying

$$
e\left(\mathcal{G}_{2}^{\prime \prime}\right) \stackrel{(3.16)}{\geqslant}\left(\frac{\eta \zeta}{2}-\frac{\rho}{d-\eta}\right) n^{2} \stackrel{(3.11)}{\geqslant} \frac{\eta \zeta}{4} n^{2}
$$

having the property that $E\left(\mathcal{G}_{2}^{\prime \prime}\right) \cap B_{\eta}=\emptyset$. Then, by [10, Proposition 1.2.2], there exists a subgraph $\mathcal{G}_{2} \subseteq \mathcal{G}_{2}^{\prime \prime}$ satisfying $\delta\left(\mathcal{G}_{2}\right) \geqslant(\eta \zeta / 4) n$, and this completes the definition of $\mathcal{G}_{2}$. We conclude this part of the definition by setting $\mathcal{N}_{2}:=V\left(\mathcal{G}_{2}\right) \cap \mathcal{N}_{2}^{\prime}$. The property $\delta\left(\mathcal{G}_{2}\right) \geqslant(\eta \zeta / 4) n$, together with the fact that all edges of $\mathcal{G}_{2}$ are of the form $\mathcal{N}_{1} \times \mathcal{N}_{2}$, implies that

$$
\left|\mathcal{N}_{2}\right| \geqslant \zeta \eta n / 4 \text {. }
$$

Next we define $\mathcal{N}_{3}(x, y)$ and $\mathcal{G}_{3}(x, y)$. For $w \in \mathcal{N}_{2}$, let $\mathcal{X}_{w}$ be the graph on $V(H)$ whose edge set is given by

$$
E\left(\mathcal{X}_{w}\right):=\left\{u z: u \in V(H), z \in N_{\mathcal{G}_{2}}(w) \subseteq \mathcal{N}_{1} \text { and }\{z, w, u\} \in E(H)\right\}
$$

Define

$$
\mathcal{N}_{3}:=\mathcal{N}_{3}(x, y):=\left\{u \in V(H): \exists w \in \mathcal{N}_{2} \text { such that } \operatorname{deg}_{\mathcal{X}_{w}}(u) \geqslant 20\right\},
$$

and let $\mathcal{G}_{3}:=\mathcal{G}_{3}(x, y)$ be the graph whose vertex set is $\mathcal{N}_{2} \cup \mathcal{N}_{3}$ and whose edge set is given by

$$
E\left(\mathcal{G}_{3}\right):=\left\{u w: u \in \mathcal{N}_{3}, w \in \mathcal{N}_{2} \text {, and } \operatorname{deg}_{\mathcal{X}_{w}}(u) \geqslant 20\right\} .
$$

Then we obtain

$$
\operatorname{deg}_{\mathcal{G}_{2}}(w) \eta n \leqslant e\left(\mathcal{X}_{w}\right) \leqslant 20 \cdot r+(n-r) \operatorname{deg}_{\mathcal{G}_{2}}(w)=20 \cdot r+\operatorname{deg}_{\mathcal{G}_{3}}(w) \operatorname{deg}_{\mathcal{G}_{2}}(w),
$$

where $r$ denotes the number of vertices $u \in V(H)$ satisfying $\operatorname{deg}_{\mathcal{X}_{w}}(u)<20$; the first inequality is owing to $E\left(\mathcal{G}_{2}\right) \cap B_{\eta}=\emptyset$, by definition of $\mathcal{G}_{2}$, and the last equality is owing to $\operatorname{deg}_{\mathcal{G}_{3}}(w)=n-r$, by definition of $r$. We may then write that

$$
\operatorname{deg}_{\mathcal{G}_{3}}(w) \geqslant \eta n-\frac{20 \cdot r}{\operatorname{deg}_{\mathcal{G}_{2}}(w)} \stackrel{\delta\left(\mathcal{G}_{2}\right) \geqslant \zeta \eta n / 4}{\geqslant} \eta n-\frac{20 \cdot r}{(\zeta \eta / 4) n} \geqslant \frac{r \leqslant n}{2} n,
$$

where the last inequality is assuming $n$ is sufficiently large. Consequently,

$$
\left|\mathcal{N}_{3}\right| \geqslant \frac{\eta}{2} n \text { and } e\left(\mathcal{G}_{3}\right) \geqslant \frac{1}{2} \sum_{w \in \mathcal{N}_{2}} \operatorname{deg}_{\mathcal{G}_{3}}(w) \geqslant\left|\mathcal{N}_{2}\right| \frac{\eta}{4} n \geqslant \frac{(3.17)}{16} n^{2}
$$

This concludes the definition of refined cascades and properties thereof. 


\subsubsection{Proof of Lemma 1.2}

With the definition of refined cascades complete, our proof of Lemma 1.2 follows closely that shown for Lemma 1.1. Indeed, the machinery of links defined for cascades carries over to refined cascades essentially verbatim.

Proof of Lemma 1.2. Given $d:=d_{1.2}, \alpha:=\alpha_{1.2}$ and $\eta:=\eta_{1.2}$ as in the premise of Lemma 1.2, set an auxiliary constant $\zeta$ satisfying (3.10), and put

$$
\rho_{1.2}(d, \alpha, \eta):=\min \left\{\eta / 4, \eta \zeta(d-\eta) / 4, d \zeta^{4} \eta^{6} / 2^{16}\right\}
$$

Let $0<\rho<\rho_{1.2}(d, \alpha, \eta)$ be fixed and let $H$ be a $(\rho, d)$-dense 3-graph satisfying $\delta(H) \geqslant \alpha\left(\begin{array}{c}n-1 \\ 2\end{array}\right)$. Let $\{x, y\}$ and $\left\{x^{\prime}, y^{\prime}\right\}$ be two disjoint pairs of vertices in $V(H)$, each having codegree at least $(d-\eta) n$ (existence of such pairs is established in (3.12) and the explanation thereafter).

By Lemma 3.1 it suffices to show that the refined cascades $\mathcal{R}_{\alpha, d, \eta}(x, y)$ and $\mathcal{R}_{\alpha, d, \eta}\left(x^{\prime}, y^{\prime}\right)$ admit an $\left(\{x, y\},\left\{x^{\prime}, y^{\prime}\right\}\right)$-link. By (3.18) and [10, Proposition 1.2.2], there exists a subgraph $F \subseteq \mathcal{G}_{3}$ satisfying $\delta(F) \geqslant\left(\zeta \eta^{2} / 16\right) n$. Then

$$
\left|V(F) \cap \mathcal{N}_{3}(x, y)\right| \geqslant \frac{\zeta \eta^{2}}{16} n
$$

Set

$$
\vec{B}:=\left\{(z, u) \in\left(V(F) \cap \mathcal{N}_{3}(x, y)\right) \times V(H): z \neq u \text { and } \operatorname{deg}_{H}(z, u, F)<d \zeta \eta^{2} n / 32\right\},
$$

and note that

$$
|\vec{B}| \leqslant \frac{\rho n^{3}}{d \zeta \eta^{2} n / 16-d \zeta \eta^{2} / 32}=\frac{32 \rho}{d \zeta \eta^{2}} n^{2},
$$

by Lemma 2.1. A symmetrical argument applied to $\mathcal{R}_{\alpha, \eta}\left(x^{\prime}, y^{\prime}\right)$ asserts that the set

$$
\vec{B}^{\prime}:=\left\{(z, u) \in\left(V\left(F^{\prime}\right) \cap N_{3}\left(x^{\prime}, y^{\prime}\right)\right) \times V(H): z \neq u \text { and } \operatorname{deg}_{H}\left(z, u, F^{\prime}\right)<d \zeta \eta^{2} n / 32\right\}
$$

satisfies $\left|\vec{B}^{\prime}\right| \leqslant 32 \rho d^{-1} \zeta^{-1} \eta^{-2} n^{2}$ as well, where $F^{\prime} \subseteq \mathcal{G}_{3}\left(x^{\prime}, y^{\prime}\right)$ is the counterpart of $F$.

The set $\left(V(F) \cap \mathcal{N}_{3}(x, y)\right) \times\left(V\left(F^{\prime}\right) \cap \mathcal{N}_{3}\left(x^{\prime}, y^{\prime}\right)\right)$ has size at least $\zeta^{2} \eta^{4} n^{2} / 2^{8}$, by (3.20); removing degenerate members (i.e. members of the form $(x, x)$ ), we retain at least $\zeta^{2} \eta^{4} n^{2} / 2^{9}$ non-degenerate members of that Cartesian product. The latter set of non-degenerate pairs we denote by $\vec{T}$. Then, for sufficiently large $n$, we have

$$
\left|\vec{T} \backslash\left(\vec{B} \cup \vec{B}^{\prime}\right)\right| \geqslant \frac{\zeta^{2} \eta^{4}}{2^{9}} n^{2}-\frac{64 \rho}{d \zeta \eta^{2}} n^{2} \stackrel{(3.19)}{\geqslant} \frac{\zeta^{2} \eta^{4}}{2^{10}} n^{2},
$$

and the lemma follows.

\subsection{A connecting lemma with no minimum degree conditions}

In Section 1.2 we mentioned that for $\therefore$-dense graphs, a connecting lemma imposing no minimum degree conditions is possible and that such a lemma is insufficient for our needs. In this section we make this precise.

Given an $n$-vertex 3-graph $H$ and a real $\beta>0$, define the sequence $H=: H_{0} \supseteq H_{1} \supseteq H_{2} \cdots$ of spanning subgraphs of $H$ as follows. At step $i$, if every pair $\{u, v\}$ of vertices of $H_{i}$ satisfies either $\operatorname{deg}_{H_{i}}(u, v) \geqslant \beta n$ or $\operatorname{deg}_{H_{i}}(u, v)=0$, then stop. Otherwise $H_{i}$ admits a pair of vertices $\{u, v\}$ satisfying $0<\operatorname{deg}_{H_{i}}(u, v)<\beta n$, in which case remove all edges of $H_{i}$ containing the pair $\{u, v\}$ and denote the resulting (spanning) subgraph of $H$ by $H_{i+1}$.

As overall there are $\left(\begin{array}{l}n \\ 2\end{array}\right)$ pairs of vertices to consider, then throughout the above process a total of at most $\beta n^{3}$ of the edges of $H$ are removed. Consequently, if $\beta$ and $H$ are such that $e(H)>\beta n^{3}$, 
then the above process terminates in a non-empty spanning subgraph of $H$, denoted $H^{(\beta)}$. The latter has the property that either $\operatorname{deg}_{H^{(\beta)}}(u, v) \geqslant \beta n$ or $\operatorname{deg}_{H^{(\beta)}}(u, v)=0$, whenever $\{u, v\}$ is a pair of vertices of $H$. Put another way, any pair of vertices $\{u, v\}$ captured by an edge of $H^{(\beta)}$ satisfies $\operatorname{deg}_{H^{(\beta)}}(u, v) \geqslant \beta n$. More concisely, one may write

$$
\begin{aligned}
& \delta_{2}^{*}\left(H^{(\beta)}\right) \\
& \quad:=\min \left\{\operatorname{deg}_{H^{(\beta)}}(u, v):\{u, v\} \in\left(\begin{array}{c}
V\left(H^{(\beta)}\right) \\
2
\end{array}\right) \text { and } \exists e \in E\left(H^{(\beta)}\right) \text { such that }\{u, v\} \subseteq e\right\} \geqslant \beta n .
\end{aligned}
$$

Pairs of vertices captured by the edges of $H^{(\beta)}$ are termed $\beta$-relevant. Such pairs of vertices, taken in $H^{(\beta)}$, can be connected in $H$ using the following lemma.

Lemma 3.2. For every $d, \beta \in(0,1]$ such that $\beta<d$, there exist an integer $n_{0}>0$ and a real $\rho_{0}>0$ such that the following holds for all $n \geqslant n_{0}$ and $0<\rho<\rho_{0}$.

Let $H$ be an $n$-vertex $(\rho, d)$-dense 3-graph and let $\{x, y\}$ and $\left\{x^{\prime}, y^{\prime}\right\}$ be two disjoint $\beta$-relevant pairs of vertices. Then there exists a 10-path in $H$ connecting $\{x, y\}$ and $\left\{x^{\prime}, y^{\prime}\right\}$.

The proof of Lemma 3.2 is that of Lemma 1.1 essentially verbatim. Let the two $\beta$-relevant pairs $\{x, y\}$ and $\left\{x^{\prime}, y^{\prime}\right\}$ per Lemma 3.2 be given. First, construct the cascades $\mathcal{C}_{\beta}(x, y)$ and $\mathcal{C}_{\beta}\left(x^{\prime}, y^{\prime}\right)$ in $H^{(\beta)}$ (instead of $H$ ), while throughout the construction of these replace every appeal to $\delta_{2}\left(H^{(\beta)}\right.$ ) (which may be zero) with an appeal to $\delta_{2}^{*}\left(H^{(\beta)}\right)$. Indeed, the construction of cascades only requires a sufficiently large minimum codegree for the pairs already captured through the edges of the cascades, and in this manner we progress from one level of the cascade to the next. Second, with these cascades constructed, note that these exist in $H$, and thus an $\left(\{x, y\},\left\{x^{\prime}, y^{\prime}\right\}\right)$-link can be found in $H$ using the very same argument seen in the proof of Lemma 1.1 for that stage.

Unfortunately we were unable to employ Lemma 3.2 in our account. Indeed, in subsequent arguments the connecting lemmas are used repeatedly to connect prescribed pairs of vertices which, although they admit a relatively large codegree, are essentially arbitrary. We were unable to determine whether these pairs are also $\beta$-relevant (for an appropriate $\beta$ ). For indeed, a pair is $\beta$-relevant if it manages to survive the clean-up procedure, so to speak, giving rise to $H^{(\beta)}$. Arbitrary pairs of vertices admitting high codegree in $H$ may of course not survive this process.

We do, however, perceive Lemma 3.2 as being relevant to the pursuit of Conjecture 1.4 and consequently mention it here.

\section{Absorbing-path lemmas}

In this section we prove Lemmas 1.3 and 1.4. At the core of these proofs stands the notion of a $\beta$-absorber, which is a variant of what is often referred to as the natural absorber as far as tight cycles in 3-graphs are concerned.

Definition 4.1. Let $H$ be a 3-graph. For $\beta>0$ and $v \in V(H)$, a quadruple $(x, y, z, w) \in V(H)^{4}$ is said to be a $(\beta, v)$-absorber if

(A.1) $\{x, y, z\},\{y, z, w\},\{v, x, y\},\{v, y, z\},\{v, z, w\} \in E(H)$,

(A.2) $\operatorname{moreover} \operatorname{deg}_{H}(x, y), \operatorname{deg}_{H}(z, w) \geqslant \beta n$.

By $\beta$-absorber we mean a $(\beta, v)$-absorber for some $v \in V(H)$.

Our proofs of both absorbing-path lemmas are modelled after the same conceptual three-step argument shown in [35]. First, a counting lemma for $(\beta, v)$-absorbers with the vertex $v$ prescribed is established; this can be seen in Lemma 4.1. Second, the aforementioned counting lemma is 
employed in a hypergeometric experiment to establish the existence of a 'small' set $\mathcal{F}$ of vertexdisjoint $\beta$-absorbers that can absorb any set of vertices that is not too 'large'; this can be seen in Lemma 4.2. Third, using the connecting lemmas, namely Lemmas 1.1 and 1.2, we 'string' the members of $\mathcal{F}$ into a single path yielding the required absorbing-path.

Lemmas 4.1 and 4.2, capturing the first two steps in the approach outlined above, are capable of handling both settings considered in Theorems 1.2 and 1.3. This is due to [32, Remark 1.4] asserting that if an $n$-vertex 3-graph $H$ admits $\delta_{2}(H)=\Omega(n)$, then $\delta(H)=\Omega\left(n^{2}\right)$.

The third step in the above plan, however, we treat separately across the two aforementioned settings. While the overall scheme of the third step is the same between the two settings, it is here that invocations to the two connecting lemmas are made. The inherent differences between these two lemmas compels (us to have) two separate treatments. We prove Lemma 1.3 in Section 4.3 and Lemma 1.4 in Section 4.4.

\subsection{A counting lemma for $\beta$-absorbers}

Let $H$ be a 3-graph and let $v \in V(H)$. We write $L_{v}:=L_{v}(H)$ to denote the link graph of $v$, that is, the graph whose vertex set is $V(H) \backslash\{v\}$ and in which two (distinct) vertices, namely $x$ and $y$, form an edge whenever $\{x, y, v\} \in E(H)$. Put

$$
L_{\beta, v}:=\left\{x y \in L_{v}: \operatorname{deg}_{H}(x, y) \geqslant \beta n\right\} .
$$

Lemma 4.1. For every $d_{4.1}, \alpha_{4.1}, \beta_{4.1} \in(0,1]$ such that $\beta_{4.1}<d_{4.1}$, there exist an integer $n_{4.1}:=$ $n_{4.1}\left(d_{4.1}, \alpha_{4.1}, \beta_{4.1}\right)$, a real $\rho_{4.1}=\rho_{4.1}\left(d_{4.1}, \alpha_{4.1}, \beta_{4.1}\right)>0$ and a real $c_{4.1}:=c_{4.1}\left(d_{4.1}, \alpha_{4.1}\right)>0$ such that the following holds for any integer $n \geqslant n_{4.1}$ and $0<\rho<\rho_{4.1}$.

Let $H$ be an $n$-vertex $\left(\rho, d_{4.1}\right)$-dense 3-graph satisfying $\delta(H) \geqslant \alpha_{4.1}\left(\begin{array}{c}n-1 \\ 2\end{array}\right)$ and let $v \in V(H)$. Then there are at least $c_{4.1} n^{4}(\beta, v)$-absorbers in $H$.

Proof. Given $\alpha:=\alpha_{4.1}, \beta:=\beta_{4.1}$ and $d:=d_{4.1}$, set

$$
\rho_{4.1}:=\min \left\{\alpha(d-\beta) / 8, d \alpha^{10} / 2^{36}\right\},
$$

let $0<\rho<\rho_{4.1}$ be fixed and let $n$ be sufficiently large. Let $H$ be an $n$-vertex $(\rho, d)_{\AA}$-dense 3-graph as in the premise and fix $v \in V(H)$.

Having $\operatorname{deg}_{H}(v) \geqslant \alpha\left(\begin{array}{c}n-1 \\ 2\end{array}\right)$ asserts that $e\left(L_{v}\right) \geqslant \alpha\left(\begin{array}{c}n-1 \\ 2\end{array}\right)$. Then, for sufficiently large $n$,

$$
e\left(L_{\beta, v}\right) \geqslant e\left(L_{v}\right)-\left|B_{\beta}\right| \stackrel{(2.3)}{\geqslant} \alpha\left(\begin{array}{c}
n-1 \\
2
\end{array}\right)-\frac{\rho}{d-\beta} n^{2} \geqslant \frac{\alpha}{4} n^{2}-\frac{\rho}{d-\beta} n^{2} \stackrel{(4.1)}{\geqslant} \alpha n^{2} / 8,
$$

where $B_{\beta}$ is as in (2.2). By [10, Proposition 1.2.2], $L_{\beta, v}$ admits a subgraph with minimum degree at least $\alpha n / 8$, implying in turn that

$$
\ell:=\left|V\left(L_{\beta, v}\right)\right| \geqslant \alpha n / 8
$$

Sidorenko's conjecture [12,39] is true for the 2-graph $P_{4}$ [3], where by $P_{4}$ we mean the path consisting of three edges and four vertices. Then, for sufficiently large $n$, there are at least

$$
(n-1)^{4}\left(\frac{2 e\left(L_{\beta, v}\right)}{n^{2}}\right)^{3} \geqslant \frac{n^{4}}{2} \cdot\left(\frac{2 \alpha}{8}\right)^{3}=\frac{\alpha^{3}}{2^{7}} n^{4}
$$

homomorphisms of $P_{4}$ into $L_{\beta, v}$. Consequently (and again assuming $n$ is sufficiently large), there is a collection $\mathcal{P}$ of at least $\alpha^{3} n^{4} / 2^{8}$ labelled copies of $P_{4}$ in $L_{\beta, v}$. 
For an ordered pair $(u, w) \in V\left(L_{\beta, v}\right) \times V\left(L_{\beta, v}\right)$, let $P_{4}(u, w)$ denote the number of members of $\mathcal{P}$ with the form $(x, u, w, y)$. Set $K:=\alpha^{3} \ell^{2} / 2^{10}$. Owing to (4.2),

$$
K \geqslant \frac{\alpha^{5}}{2^{16}} n^{2}
$$

Put

$$
\vec{X}:=\left\{(u, w) \in V\left(L_{\beta, v}\right) \times V\left(L_{\beta, v}\right): P_{4}(u, w)<K\right\}
$$

and let

$$
\vec{Y}:=V\left(L_{\beta, v}\right) \times V\left(L_{\beta, v}\right) \backslash \vec{X}
$$

Then

$$
\frac{\alpha^{3}}{2^{8}} \ell^{4} \leqslant \frac{\alpha^{3}}{2^{8}} n^{4} \leqslant|\mathcal{P}|=\sum_{(u, w) \in V\left(L_{\beta, v}\right)^{2}} P_{4}(u, w) \leqslant|\vec{X}| \cdot K+\left(\ell^{2}-|\vec{X}|\right) \ell^{2} .
$$

Isolating $|\vec{X}|$, one arrives at

$$
|\vec{X}| \leqslant \frac{\left(1-\alpha^{3} / 2^{8}\right) \ell^{4}}{\ell^{2}-K}=\frac{1-\alpha^{3} / 2^{8}}{1-\alpha^{3} / 2^{10}} \ell^{2} \leqslant\left(1-\frac{\alpha^{3}}{2^{10}}\right) \ell^{2} .
$$

As a result, we attain

$$
|\vec{Y}| \geqslant \frac{\alpha^{3}}{2^{10}} \ell^{2} \stackrel{(4.2)}{\geqslant} \frac{\alpha^{5}}{2^{16}} n^{2}
$$

In preparation for two applications of Lemma 2.1, we define three graphs, namely $G_{1}, G_{2}$ and $G_{3}$, edges of which collectively capture the members of $\mathcal{P}$. Lemma 2.1 is then applied to $G_{1}$ and $G_{3}$ (along with additional parameters defined below); the resulting estimates attained from these two applications of the lemma are then used to analyse $G_{2}$. $\vec{Y}$, set

Set $G_{2}:=\left(V\left(L_{\beta, v}\right), Y\right)$, where $Y$ denotes the set of unordered pairs underlying $\vec{Y}$. For $(u, w) \in$

$$
\vec{A}_{(u, w)}:=\left\{(a, u):(a, u, w, b) \in \mathcal{P} \text { for some } a, b \in V\left(L_{\beta, v}\right)\right\}
$$

and set

$$
\vec{B}_{(u, w)}:=\left\{(w, b):(a, u, w, b) \in \mathcal{P} \text { for some } a, b \in V\left(L_{\beta, v}\right)\right\}
$$

Define

$$
G_{1}:=\left(V\left(L_{\beta, v}\right), \bigcup_{(u, w) \in \vec{Y}} A_{(u, w)}\right) \text { and } G_{3}:=\left(V\left(L_{\beta, v}\right), \bigcup_{(u, w) \in \vec{Y}} B_{(u, w)}\right),
$$

where $A_{(u, w)}$ and $B_{(u, w)}$ are the sets of unordered pairs underlying $\vec{A}_{(u, w)}$ and $\vec{B}_{(u, w)}$, respectively. The graphs $G_{1}, G_{2}, G_{3}$ are not necessarily edge-disjoint.

Define the sets of vertices

$$
\begin{aligned}
U & :=\left\{u \in V\left(L_{\beta, v}\right):(a, u, w, b) \in \mathcal{P} \text { for some } a, w, b \in V\left(L_{\beta, v}\right) \text { and }(u, w) \in \vec{Y}\right\}, \\
W & :=\left\{w \in V\left(L_{\beta, v}\right):(a, u, w, b) \in \mathcal{P} \text { for some } a, u, b \in V\left(L_{\beta, v}\right) \text { and }(u, w) \in \vec{Y}\right\} .
\end{aligned}
$$

Observe that $U \subseteq V\left(G_{1}\right)$ and that $W \subseteq V\left(G_{3}\right)$.

For $(u, w) \in \overline{\vec{Y}}$, observe that $\left|\vec{A}_{(u, w)}\right|,\left|\vec{B}_{(u, w)}\right| \geqslant \alpha^{5} n / 2^{16}$. For if one of these sets, say $\vec{A}_{(u, w)}$, violates this inequality, then

$$
P_{4}(u, w) \leqslant\left|\vec{A}_{(u, w)}\right| \operatorname{deg}_{L_{\beta, v}}(w)<\frac{\alpha^{5}}{2^{16}} n \cdot n \stackrel{(4.3)}{\leqslant} K,
$$


in contradiction to $(u, w) \in \vec{Y}$. Consequently, $\operatorname{deg}_{G_{1}}(u), \operatorname{deg}_{G_{3}}(w) \geqslant \alpha^{5} n / 2^{16}$ for every $u \in U$ and every $w \in W$, respectively.

Set

$$
\begin{aligned}
\vec{B}_{U} & :=\left\{(u, w) \in U \times W: \operatorname{deg}_{H}\left(u, w, G_{1}\right)<d \alpha^{5} n / 2^{17}\right\}, \\
\vec{B}_{W} & :=\left\{(u, w) \in U \times W: \operatorname{deg}_{H}\left(w, u, G_{3}\right)<d \alpha^{5} n / 2^{17}\right\} .
\end{aligned}
$$

Lemma 2.1, applied to $G_{1}, U, \vec{B}_{U}$ and to $G_{3}, W, \vec{B}_{W}$, asserts that

$$
\left|\vec{B}_{U}\right|,\left|\vec{B}_{W}\right| \leqslant \frac{2^{17} \rho}{d \alpha^{5}} n^{2} .
$$

Owing to (4.4), $e\left(G_{2}\right) \geqslant \alpha^{5} n^{2} / 2^{17}$ holds. This fact, together with the estimates seen in (4.5), implies that $G_{2}$ admits at least

$$
e\left(G_{2}\right)-\left|\vec{B}_{U}\right|-\left|\vec{B}_{W}\right| \stackrel{(4.4)}{\geqslant} \frac{\alpha^{5}}{2^{17}} n^{2}-\frac{2^{18} \rho}{d \alpha^{5}} n^{2} \stackrel{(4.1)}{\geqslant} \frac{\alpha^{5}}{2^{18}} n^{2}
$$

unordered pairs $\{u, w\} \in E\left(G_{2}\right) \subseteq E\left(L_{\beta, v}\right)$ with $u \in U$ and $w \in W$ such that

$$
\operatorname{deg}_{H}\left(u, w, G_{1}\right), \quad \operatorname{deg}_{H}\left(w, u, G_{3}\right) \geqslant d \alpha^{5} n / 2^{17} .
$$

Call these pairs in $E\left(G_{2}\right)$ good. ${ }^{\mathrm{g}}$

Let $(u, w) \in U \times W$ be good. At least ${ }^{\mathrm{h}} \operatorname{deg}_{H}\left(u, w, G_{1}\right)-1$ neighbours $a$ of $u$ in $G_{1}$ satisfy $a \neq w$. Each such neighbour $a$ of $u$ gives rise to a triple $(a, u, w)$ with the property that $a u \in E\left(L_{\beta, v}\right)$ so that $\operatorname{deg}_{H}(a, u) \geqslant \beta n$. The triple $(a, u, w)$ extends to at least ${ }^{\mathrm{i}} \operatorname{deg}_{H}\left(w, u, G_{3}\right)-2$ quadruples $(a, u, w, b)$ satisfying $b \notin\{a, u\}$ and $w b \in E\left(L_{\beta, v}\right)$ so that $\operatorname{deg}_{H}(w, b) \geqslant \beta n$ holds. Any quadruple thus formed defines a $(\beta, v)$-absorber.

It follows that for sufficiently large $n$, a single good pair $(u, w)$ gives rise to at least

$$
\left(\frac{d \alpha^{5}}{2^{17}} n-1\right)\left(\frac{d \alpha^{5}}{2^{17}} n-2\right) \geqslant \frac{d^{2} \alpha^{10}}{2^{35}} n^{2}
$$

$(\beta, v)$ - absorbers. Ranging over all good pairs $(u, w)$, we attain at least

$$
\frac{\alpha^{5}}{2^{18}} n^{2} \cdot \frac{d^{2} \alpha^{10}}{2^{35}} n^{2}=\frac{d^{2} \alpha^{15}}{2^{53}} n^{4}
$$

$(\beta, v)$-absorbers overall, concluding the proof of the lemma.

\subsection{A 'small' set of $\beta$-absorbers}

Let $H$ be a 3-graph. For $v \in V(H)$ and $\beta>0$, let $\mathcal{A}_{\beta, v}$ denote the set of $(\beta, v)$-absorbers in $H$.

Lemma 4.2. For every $d_{4.2}, \alpha_{4.2}, \beta_{4.2}, \phi_{4.2} \in(0,1]$ such that $\beta_{4.2}<d_{4.2}$, there exist an integer $n_{4.2}:=n_{4.2}\left(d_{4.2}, \alpha_{4.2}, \beta_{4.2}, \phi_{4.2}\right)$, and reals $\rho_{4.2}:=\rho_{4.2}\left(d_{4.2}, \alpha_{4.2}, \beta_{4.2}\right)>0$ and $\eta_{4.2}:=$ $\eta_{4.2}\left(d_{4.2}, \alpha_{4.2}, \phi_{4.2}\right)>0$ such that the following holds whenever $n \geqslant n_{4.2}$ and $\rho<\rho_{4.2}$.

Let $H$ be an $n$-vertex $\left(\rho, d_{4.2}\right)$-dense 3-graph satisfying $\delta(H) \geqslant \alpha_{4.2}\left(\begin{array}{c}n-1 \\ 2\end{array}\right)$. Then there exists a set $\mathcal{F}$ of vertex-disjoint $\beta$-absorbers such that

(F.1) $|\mathcal{F}| \leqslant \phi_{4.2} n$,

(F.2) for every $v \in V(H),\left|\mathcal{A}_{\beta_{4.2}, v} \cap \mathcal{F}\right| \geqslant \eta_{4.2} n$.

${ }^{\mathrm{g}}$ As good pairs arise from edges of a simple graph these are non-degenerate.

${ }^{\text {h}}$ Strictly speaking, $\operatorname{deg}_{H}\left(u, w, G_{1}\right)-1$ can be replaced with $\operatorname{deg}_{H}\left(u, w, G_{1}\right)$ as $\{a, u, w\} \in E(H)$.

${ }^{\text {iS }}$ trictly speaking, $\operatorname{deg}_{H}\left(w, u, G_{3}\right)-2$ can be replaced with $\operatorname{deg}_{H}\left(w, u, G_{3}\right)-1$ as $\{u, w, b\} \in E(H)$. 
Proof. Given

$$
d:=d_{4.2}, \quad \alpha:=\alpha_{4.2}, \quad \beta:=\beta_{4.2} \quad \text { and } \quad \phi:=\phi_{4.2}
$$

as in the premise, set $\rho_{4.2}:=\rho_{4.1}(d, \alpha, \beta)$. In addition, define the auxiliary constants

$$
c:=c_{4.1}(d, \alpha) \quad \text { and } \quad \gamma:=\min \left\{\frac{c}{4 \cdot 7}, \frac{\phi}{2}\right\} .
$$

Finally, set $\eta_{4.2}:=c \gamma / 4$.

Fix $0<\rho<\rho_{4.2}$. Let $H$ be a $(\rho, d) \ldots$-dense 3 -graph as in the premise. Then $\left|\mathcal{A}_{\beta, v}\right| \geqslant c n^{4}$ for every $v \in V(H)$, by Lemma 4.1. Let $\mathcal{F}^{\prime}$ be a set of quadruples where each quadruple in $V(H)^{4}$ is put in $\mathcal{F}^{\prime}$ independently at random with probability $\gamma n^{-3}$. Then $\mathbb{E}\left[\left|\mathcal{F}^{\prime}\right|\right]=\gamma n$. Chernoff's inequality $[18$, equation (2.9)] then yields that

$$
\left|\mathcal{F}^{\prime}\right| \leqslant 2 \gamma n \leqslant \phi n
$$

with probability $1-o(1)$. Furthermore, for every vertex $v$,

$$
\mathbb{E}\left[\left|\mathcal{A}_{\beta, v} \cap \mathcal{F}^{\prime}\right|\right] \geqslant c n^{4} \gamma n^{-3}=c \gamma n .
$$

Chernoff's inequality [18, equation (2.9)] and the union bound yield that

$$
\left|\mathcal{A}_{\beta, v} \cap \mathcal{F}^{\prime}\right| \geqslant c \gamma n / 2 \text { for every } v \in V(H)
$$

with probability $1-o(1)$.

Let $I:=I\left(\mathcal{F}^{\prime}\right)$ denote the number of pairs of members of $\mathcal{F}^{\prime}$ meeting one another. For sufficiently large $n$, the total number of pairs of intersecting quadruples taken in $V(H)$ is at most

$$
\left(\begin{array}{l}
4 \\
1
\end{array}\right) n^{4} \cdot n^{3}+\left(\begin{array}{l}
4 \\
2
\end{array}\right)(2 !) n^{4} \cdot n^{2}+\left(\begin{array}{l}
4 \\
3
\end{array}\right)(3 !) n^{4} \cdot n \leqslant 6 n^{7} .
$$

Then

$$
\mathbb{E}[|I|] \leqslant 6 n^{7} \cdot\left(\gamma n^{-3}\right)^{2} \leqslant 6 \gamma^{2} n .
$$

Markov's inequality now implies that

$$
|I|<7 \gamma^{2} n
$$

with positive probability.

It follows that an $\mathcal{F}^{\prime}$ satisfying (4.7), (4.8) and (4.9) exists. Fix one such $\mathcal{F}^{\prime}$. Define $\mathcal{F}$ to be the set of quadruples attained from $\mathcal{F}^{\prime}$ by first removing all quadruples that do not $\beta$-absorb any $v$, and second, from each intersecting pair of quadruples, removing one of the members of that pair. Property (F.1) trivially holds for $\mathcal{F}$. To see that (F.2) holds for $\mathcal{F}$, note that, for every $v \in V(H)$,

$$
\left|\mathcal{A}_{\beta, v} \cap \mathcal{F}\right| \geqslant c \gamma n / 2-7 \gamma^{2} n \stackrel{(4.6)}{\geqslant} c \gamma n / 4=\eta_{4.2} n
$$

whenever $n$ is sufficiently large.

\subsection{Proof of Lemma 1.3: 2-degree setting}

With Lemmas 4.1 and 4.2 established, we are ready to prove Lemma 1.3. All that remains is to 'string' the members of $\mathcal{F}$ (from Lemma 4.2) into a single path and prove the absorption capabilities of the resulting path. Given a quadruple $(x, y, z, w)$, we refer to $(x, y)$ and $(z, w)$ as the front and rear end-pair of the quadruple respectively.

Proof of Lemma 1.3. Let $d:=d_{1.3}, \alpha:=\alpha_{1.3}, \beta:=\beta_{1.3}<\min \{\alpha, d\}$ be given. Set

$$
\kappa:=\kappa_{1.3}:=\beta / 2 \text { and } \mu:=\mu_{1.3}:=\eta_{4.2}(d, \alpha, \beta / 20) \text {. }
$$


To be clear, the definition of $\mu$ appeals to that of $\eta_{4.2}$. The latter requires a value for $\phi_{4.2}$ be set; here we take $\phi_{4.2}=\beta / 20$. Set

$$
\rho_{1.3}:=\min \left\{\rho_{4.2}(d, \alpha, \beta, \beta / 20), \quad \rho_{1.1}(d, \beta-\kappa) \cdot(1-\kappa)^{3} / 2\right\} .
$$

Let $0<\rho<\rho_{1.3}$ be fixed, let $n$ be a sufficiently large integer and let $H$ be an $n$-vertex $(\rho, d) \therefore$-dense 3-graph with $\delta_{2}(H) \geqslant \alpha(n-2)$.

Let $\mathcal{F}$ denote the set of $\beta$-absorbers, the existence of which in $H$ is assured by Lemma 4.2 applied with $\alpha_{4.2}=\alpha, d_{4.2}=d, \beta_{4.2}=\beta, \phi_{4.2}=\beta / 20$, and owing to $\rho<\rho_{4.2}(d, \alpha, \beta, \beta / 20)$, by (4.11). Fix an arbitrary ordering of the members of $\mathcal{F}$, namely $F_{1}, F_{2}, \ldots, F_{r}$, where $r:=|\mathcal{F}| \leqslant$ $\beta n / 20$, by (F.1). In what follows, we prove that a path $A$ of the form

$$
F_{1} \circ P_{1} \circ \cdots \circ F_{r-1} \circ P_{r-1} \circ F_{r}
$$

exists in $H$, where each $P_{i}$ is a 10-path connecting the rear end-pair of $F_{i}$ with the front end-pair of $F_{i+1}$; we use $\circ$ to denote path concatenations along these pairs. If such a path $A$ were to exist, then it would form a $(\beta, \mu, \kappa)$-absorbing-path. To see this, first observe that

$$
|V(A)|=4 r+6(r-1) \leqslant 10 r \leqslant \beta n / 2 \stackrel{(4.10)}{=} \kappa n .
$$

Then observe that (F.2), together with a standard greedy argument (see e.g. [35, Claim 2.6]), asserts that such a path $A$ would form a $\mu n$-absorbing-path. Finally observe that the ends of such a path $A$ would have codegree at least $\beta n$ for, indeed, $\beta<\alpha$, and $\delta_{2}(H) \geqslant \alpha(n-2)$ (here we utilize the fact that $n$ is sufficiently large).

It remains to establish the existence of the aforementioned path. This we do inductively as follows. Put $A_{1}:=F_{1}$. Suppose that the (partial) path

$$
A_{i}:=F_{1} \circ P_{1} \circ \cdots \circ F_{i-1} \circ P_{i-1} \circ F_{i}
$$

has been defined for some $i \in[r-1]$. We define $A_{i+1}$ as follows. Set

$$
V_{i}:=\left(V(H) \backslash\left(V\left(A_{i}\right) \cup V(\mathcal{F})\right)\right) \cup\{a, b, c, d\},
$$

where $(a, b)$ is the rear end-pair of $F_{i}$ and $(c, d)$ is the front end-pair of $F_{i+1}$. The next two claims verify that Lemma 1.1 can be applied to $H\left[V_{i}\right]$ in order to connect $(a, b)$ with $(c, d)$ via $H\left[V_{i}\right]$.

Claim 2. Let $i \in[r]$. Then $H\left[V_{i}\right]$ is $\left(\rho^{\prime}, d\right)$-dense for some $\rho^{\prime}<\rho_{1.1}(d, \beta / 2)$.

Proof. As $\left|V\left(A_{i}\right) \cup V(\mathcal{F})\right| \leqslant \kappa n$, it follows that $\left|V_{i}\right| \geqslant(1-\kappa) n$ for every $i \in[r]$. Fix $\vec{G}_{1}, \vec{G}_{2} \subseteq V_{i} \times$ $V_{i}$, and note that

$$
\begin{aligned}
e_{H\left[V_{i}\right]}\left(\vec{G}_{1}, \vec{G}_{2}\right) & \geqslant d\left|\mathcal{P}_{2}\left(\vec{G}_{1}, \vec{G}_{2}\right)\right|-\rho n^{3} \\
& \stackrel{(4.11)}{\geqslant} d\left|\mathcal{P}_{2}\left(\vec{G}_{1}, \vec{G}_{2}\right)\right|-\rho_{1.1}(d, \beta-\kappa) \frac{(1-\kappa)^{3}}{2} n^{3} \\
& \geqslant d\left|\mathcal{P}_{2}\left(\vec{G}_{1}, \vec{G}_{2}\right)\right|-\frac{\rho_{1.1}(d, \beta-\kappa)}{2}\left|V_{i}\right|^{3} .
\end{aligned}
$$

Owing to $\kappa=\beta / 2$, the claim follows.

Claim 3. Let $i \in[r]$. Then $\delta_{2}\left(H\left[V_{i}\right]\right) \geqslant \beta n / 2$.

Proof. Owing to $\beta<\alpha, \delta_{2}(H) \geqslant \alpha(n-2)$, and $n$ being sufficiently large, we may write

$$
\delta_{2}\left(H\left[V_{i}\right]\right) \geqslant \alpha(n-2)-\kappa n=(\alpha-\kappa) n-2 \alpha \stackrel{\alpha>\beta}{\geqslant}(\beta-\kappa) \stackrel{(4.10)}{=} \beta n / 2 .
$$

Lemma 1.1, applied to $H\left[V_{i}\right]$ with $\alpha_{1.1}=\beta / 2$ and $d_{1.1}=d$, asserts that any two pairs of vertices in $H\left[V_{i}\right]$ can be connected via a 10-path in $H\left[V_{i}\right]$. This in particular holds for the pairs $(a, b)$ and 
$(c, d)$. The path $P_{i+1}$, as defined above, exists and consequently $A_{i+1}$ as well. This completes the proof of the existence of $A$ and thus concludes the proof of the lemma.

\subsection{Proof of Lemma 1.4: 1-degree setting}

Given $\alpha:=\alpha_{1.4}, d:=d_{1.4}$ and $\eta:=\eta_{1.4}$ as in the premise of the lemma, choose two constants $0<\zeta \ll \kappa$ such that

$$
\kappa \ll \min \left\{\alpha, d, \eta, \frac{\alpha+d-1}{2(d+1)+1}\right\}, \quad \zeta+\kappa \ll g(\alpha-\kappa, d),
$$

where $g(\cdot)$ is as defined in (1.3). The first constraint being our prerogative, we explain the validity of the second. Owing to $\zeta \ll \kappa$, it suffices to argue that there exists a choice of $\kappa$ satisfying

$$
2 \kappa \ll g(\alpha-\kappa, d) \stackrel{(1.3)}{=} \min \left\{\alpha-\kappa, d, \frac{\alpha-\kappa+d-1}{d+1}\right\} .
$$

The first term in the minimization entails having to require $\kappa \ll \alpha$. The second term imposes $\kappa \ll d$. The third, and final, term requires

$$
\kappa \ll \frac{\alpha+d-1}{2(d+1)+1} .
$$

We remark that the condition $\kappa \ll \eta$ plays no role in the proof. It is, however, mandated in order to accommodate a subsequent application of Lemma 1.4 in the proof of Theorem 1.3 in Section 6.2.

With $\zeta$ and $\kappa$ fixed, define

$$
\kappa_{1.4}:=\kappa \quad \text { and } \quad \mu:=\mu_{1.4}:=\eta_{4.2}(d, \alpha, \kappa / 10) .
$$

To be clear, the definition of $\mu$ entails setting $\phi_{4.2}:=\kappa / 10$. Set

$$
\rho_{1.4}:=\min \left\{\rho_{4.2}(\alpha, d, d-\zeta, \kappa / 10), \quad 2^{-1}(1-\kappa)^{3} \rho_{1.2}(d, \alpha-\kappa, \zeta+2 \kappa)\right\} .
$$

Let $0<\rho<\rho_{1.4}$ be fixed and let $H$ be a $(\rho, d)$-dense 3 -graph satisfying $\delta(H) \geqslant \alpha_{1.4}\left(\begin{array}{c}n-1 \\ 2\end{array}\right)$ be given. Let $\mathcal{F}$ be a set of $(d-\zeta)$-absorbers, the existence of which is assured by Lemma 4.2 applied with $\alpha_{4.2}=\alpha, d_{4.2}=d, \beta_{4.2}=d-\zeta$ and $\phi_{4.2}=\kappa / 10$, and also owing to $\rho<\rho_{4.2}(\alpha, d, d-$ $\zeta, \kappa / 10)$ per (4.18). As in the proof of Lemma 1.3, we seek to establish the existence of a path $A$ of the form (4.14). If such a path $A$ were to exist, then it would form a $(\beta-\kappa, \mu, \kappa)$-absorbingpath. Indeed, owing to $r:=|\mathcal{F}| \leqslant \kappa n / 10$ its length would be at most $\kappa n$, by (4.13); it would be $\mu n$-absorbing, by [35, Claim 2.6]; its ends would have codegree at least $(d-\eta) n$ as these arise from $(d-\zeta)$-absorbers in $\mathcal{F}$ and $\zeta \ll \eta$.

It remains to establish the existence of $A$. Let $A_{i}$ and $V_{i}$ be as defined in (4.14) and (4.15), respectively. It suffices to prove that the pairs $(a, b)$ and $(c, d)$ (per the definition of $V_{i}$ ) can be connected via a 10-path passing through $H\left[V_{i}\right]$. This we accomplish using Lemma 1.2. Hence it remains to prove that for every $i \in[r], H\left[V_{i}\right]$ adheres to the premise of that lemma. The following claims verify this.

Starting with the $\therefore$-denseness of $H\left[V_{i}\right]$, note that (4.18), the observation that $\left|V_{i}\right| \geqslant(1-\kappa) n$, and an argument identical to that seen in (4.16) establish the following.

Claim 4. Let $i \in[r]$. Then $H\left[V_{i}\right]$ is $\left(\rho^{\prime}, d\right) \ldots$-dense for some $\rho^{\prime}<\rho_{1.2}(d, \alpha-\kappa, \zeta+2 \kappa)$.

Claim 5. Let $i \in[r]$. Then

$$
\delta\left(H\left[V_{i}\right]\right) \geqslant(\alpha-\kappa)\left(\begin{array}{c}
\left|V_{i}\right|-1 \\
2
\end{array}\right)
$$


Proof. Start by observing that

$$
\delta\left(H\left[V_{i}\right]\right) \geqslant \delta(H)-\left|V\left(A_{i}\right) \cup V(\mathcal{F})\right| \cdot n,
$$

where the last term on the right-hand side accounts for all pairs involving vertices from $V\left(A_{i}\right) \cup$ $V(\mathcal{F})$. Owing to (4.13) and relying on $n$ being sufficiently large, we may write

$$
\begin{aligned}
\delta\left(H\left[V_{i}\right]\right) & \geqslant \alpha\left(\begin{array}{c}
n-1 \\
2
\end{array}\right)-\kappa n^{2} \\
& \geqslant \alpha \frac{n^{2}}{2}-\kappa n^{2}-\alpha \frac{n}{2} \\
& =(\alpha-\kappa / 2) \frac{n^{2}}{2}-\alpha \frac{n}{2} \\
& \geqslant(\alpha-\kappa) \frac{n^{2}}{2} \\
& \geqslant(\alpha-\kappa)\left(\begin{array}{c}
\left|V_{i}\right|-1 \\
2
\end{array}\right) .
\end{aligned}
$$

Having set $\kappa \ll \alpha+d-1$ in (4.17), this implies that $(\alpha-\kappa)+d>1$ holds. This, coupled with the condition $\zeta+\kappa \ll g(\alpha-\kappa, d)$, also set in (4.17), implies that any two disjoint pairs of vertices having codegree at least $(d-\zeta-\kappa) n$ can be connected in $H\left[V_{i}\right]$ via a 10 -path, by Lemma 1.2. In $H\left[V_{i}\right]$, both pairs $(a, b)$ and $(c, d)$ have codegree at least $(d-\zeta-\kappa) n$ and are thus connectable in this fashion. The existence of $A$ is established. This concludes the proof of the lemma.

\section{The path-cover lemma}

In this section we prove our path-cover lemma, namely Lemma 1.5. Our proof of this lemma employs the weak regularity lemma stated below in Lemma 5.1. In Section 5.2 we provide an alternative proof of Lemma 1.5 for graphs equipped with the notion of $\bullet$-denseness; the latter notion is a stronger notion than that of 1 -set-denseness assumed in Lemma 1.5 . If $\bullet \bullet$-denseness is assumed, then the regularity lemma is no longer needed, giving rise to a much shorter proof.

\subsection{Path covers in 1-set-dense 3-graphs}

A 3-graph $H$ is said to be $t$-partite if there is a vertex partition $V(H)=V_{1} \dot{\cup} V_{2} \dot{\cup} \ldots \dot{\cup} V_{t}$ such that each edge $e \in E(H)$ satisfies $\left|e \cap V_{i}\right| \leqslant 1$ whenever $i \in[t]$. A $t$-partite $H$ is said to be equitable if its $t$-partition satisfies $\left|V_{1}\right| \leqslant\left|V_{2}\right| \leqslant \cdots \leqslant\left|V_{t}\right| \leqslant\left|V_{1}\right|+1$. We also refer to the partition itself as equitable. An $n$-vertex 3-partite 3-graph $H$ with an underlying partition $V(H)=X \dot{\cup} Y \dot{\cup} Z$ is said to be $\varepsilon$-regular if

$$
e_{H}\left(X^{\prime}, Y^{\prime}, Z^{\prime}\right)=\frac{e_{H}(X, Y, Z)}{|X||Y||Z|}\left|X^{\prime}\right|\left|Y^{\prime}\right|\left|Z^{\prime}\right| \pm \varepsilon n^{3}
$$

for every $X^{\prime} \subseteq X, Y^{\prime} \subseteq Y$ and $Z^{\prime} \subseteq Z$. If only the lower bound seen at (5.1) is upheld by $H$, then we refer to such an $H$ as $\varepsilon$-lower-regular. If in addition $e_{H}(X, Y, Z) /|X||Y||Z| \geqslant d$, then $H$ is called $(\varepsilon, d)$-regular or $(\varepsilon, d)$-lower-regular, respectively. The following result is a commonly known generalization of the main result of [40].

Lemma 5.1 (weak-regularity lemma for 3-graphs [40]). For every $\varepsilon_{5.1}>0$ and integer $t_{5.1}$, there exist integers $n_{5.1}$ and $T_{5.1}$ such that the following holds whenever $n \geqslant n_{5.1}$. 
Let $H$ be an n-vertex 3-graph. Then there exists an integer $t$ satisfying $t_{5.1} \leqslant t \leqslant T_{5.1}$ and an equitable partition $V(H)=V_{1} \dot{\cup} V_{2} \dot{\cup} \cdots \dot{U} V_{t}$ such that, for all but at most $\varepsilon t^{3}$ triples $i, j, k \in[t]$, the sets $V_{i}, V_{j}, V_{k}$ induce an $\varepsilon_{5.1}$-regular 3-partite 3-graph denoted $H\left[V_{i}, V_{j}, V_{k}\right]$.

Given a 3-graph $H$, regularized per Lemma 5.1, and a real $d>0$, define $R_{d}:=R_{d}(H)$ to denote the 3-graph whose vertices are the clusters (i.e. sets) $\left(V_{i}\right)_{i \in[t]}$ and whose edges are the triples $\left\{V_{i}, V_{j}, V_{k}\right\}, i, j, k \in[t]$, such that $H\left[V_{i}, V_{j}, V_{k}\right]$ is $(\varepsilon, d)$-regular. It will be convenient to identify $V\left(R_{d}\right)$ with $[t]:=\{1, \ldots, t\}$. Given $X \subseteq V\left(R_{d}\right)$ define $\cup X:=\bigcup_{i \in X} V_{i}$. An edge $e \in E(H)$ is said to be crossing with respect to $X$ if there are three clusters $V_{i}, V_{j}, V_{k}$ captured by $X$ such that

$$
1=\left|e \cap V_{i}\right|=\left|e \cap V_{j}\right|=\left|e \cap V_{k}\right| \text {. }
$$

Lemma 5.2 (path-packing lemma [36, Claim 4.2]). For all $0<\varepsilon<d<1$, every $(\varepsilon, d)$-lowerregular 3-partite equitable 3-graph $H$ on $n$ vertices, with $n$ a sufficiently large integer, contains a family $\mathcal{P}$ of vertex disjoint-paths such that for each $P \in \mathcal{P}$ we have

$$
|V(P)| \geqslant \varepsilon(d-\varepsilon) n / 3 \text { and } \sum_{P \in \mathcal{P}}|V(P)| \geqslant(1-2 \varepsilon) n .
$$

The following is a triviality whose proof is included for completeness.

Lemma 5.3. For every $d_{5.3}>0$ and $\zeta_{5.3}>0$, there exist an integer $n_{5.3}:=n_{5.3}\left(d_{5.3}, \zeta_{5.3}\right)>0$ and $a$ real $\rho_{5.3}\left(d_{5.3}, \zeta_{5.3}\right)>0$ such that the following holds whenever $n \geqslant n_{5.3}$ and $0<\rho<\rho_{5.3}$.

Let $H$ be an $n$-vertex $\left(\rho, d_{5.3}\right)$-dense 3 -graph. Then $H$ admits a matching covering all but at most $\max \left\{2, \zeta_{5.3} n\right\}$ vertices.

Proof. Given $d:=d_{5.3}$ and $\zeta:=\zeta_{5.3}$, set $\rho_{5.3}:=d \cdot \zeta^{3} / 27$. Let $0<\rho<\rho_{5.3}$, let $n$ be sufficiently large and let $H$ be an $n$-vertex 3 -graph as in the premise. Let $M$ be a maximum matching in $H$. Let $X:=V(H) \backslash V(M)$ denote the set of vertices not covered by the members of $M$. If $|X| \leqslant 2$, then the claim follows. Assume then that $|X| \geqslant 3$. In this case $e_{H}(X)=0$ by the maximality of $M$. Then

$$
d\left(\begin{array}{c}
|X| \\
3
\end{array}\right)-\rho n^{3} \leqslant e_{H}(X) \leqslant 0 .
$$

Consequently

$$
\frac{d}{27}|X|^{3} \leqslant \rho n^{3}
$$

Assuming that $|X|>\zeta n$, we arrive at

$$
\zeta n<|X| \leqslant\left(27 \cdot \rho d^{-1}\right)^{1 / 3} n,
$$

contradicting $\rho<\rho_{5.3}$ chosen at the outset. Consequently, in this case $|X| \leqslant \zeta n$ must hold.

We are now ready to prove our path-cover lemma, namely Lemma 1.5.

Proof of Lemma 1.5. Given $d:=d_{1.5}$ and $\zeta:=\zeta_{1.5}$ let $\rho^{\prime}:=\rho_{5.3}(d / 2, \zeta / 12)$ and set

$$
t_{\text {reg }}:=\max \left\{8 / \rho^{\prime}, 8 / \zeta\right\}, \quad d^{\prime}:=\rho^{\prime} / 4, \quad \varepsilon_{\text {reg }}:=\min \left\{d^{\prime} / 2, \zeta / 24\right\} .
$$

In addition, set

$$
\rho_{1.5}:=\rho^{\prime} / 4 \quad \text { and } \quad \ell_{1.5}:=\frac{T_{5.1}\left(\varepsilon_{\mathrm{reg}}, t_{\mathrm{reg}}\right)}{\varepsilon_{\mathrm{reg}}\left(d^{\prime}-\varepsilon_{\mathrm{reg}}\right)} .
$$

Let $n$ be sufficiently large, let $\rho<\rho_{1.5}$ and let $H$ be an $n$-vertex $(\rho, d)$-dense 3-graph. 
Let $R_{d^{\prime}}:=R_{d^{\prime}}(H)$ denote the reduced graph of $H$ obtained after regularizing $H$ using the weakregularity lemma, namely Lemma 5.1, applied with $\varepsilon_{5.1}=\varepsilon_{\text {reg }}$ and $t_{5.1}=t_{\text {reg. }}$. Let $t:=\left|V\left(R_{d^{\prime}}\right)\right|$ and identify $V\left(R_{d^{\prime}}\right)$ with $[t]$.

Claim 6. $R_{d^{\prime}}$ is $\left(\rho^{\prime}, d / 2\right)$-dense.

Proof. Fix $X \subseteq V\left(R_{d^{\prime}}\right)$ and let $C_{X}$ denote the number of edges of $H$ which are crossing with respect to $X$ and that lie in $\left(\varepsilon_{\text {reg }}, d^{\prime}\right)$-regular triples $H\left[V_{i}, V_{j}, V_{k}\right]$, where the sets $V_{i}, V_{j}, V_{k}$ are taken from the underlying regularity partition. Then

$$
e_{R_{d^{\prime}}}(X) \geqslant C_{X} / 2(n / t)^{3} \text {. }
$$

The factor 2 appearing here is incurred in order to cope with the the fact that cluster sizes are in the set $\{n / t, n / t+1\}$; we use the fact that for sufficiently large $n, 2(n / t)^{3} \geqslant(n / t+1)^{3}$ holds.

Observe that

$$
C_{X} \geqslant e_{H}(\cup X)-|X| \cdot 2(n / t)^{3}-|X|^{2} \cdot 2(n / t)^{3}-\varepsilon_{\mathrm{reg}} t^{3} \cdot 2(n / t)^{3}-|X|^{3} d^{\prime} \cdot 2(n / t)^{3} .
$$

Indeed, the second and third terms on the right-hand side arise from the removal of all edges that have at least two of their vertices in the same cluster captured by $X$ from $E(H[\cup X])$. The fourth term on the right-hand side arises from the removal of all (crossing) edges found in $\varepsilon_{\text {reg }}{ }^{-}$ irregular triples of clusters. Finally, the last term on the right-hand side arises from the removal of all (crossing) edges found in triples of clusters whose edge density is at most $d^{\prime}$.

As $|X| \leqslant t$, we arrive at

$$
e_{d^{\prime}}(X) \geqslant \frac{e_{H}(\cup X)}{2(n / t)^{3}}-t-t^{2}-\varepsilon_{\mathrm{reg}} t^{3}-d^{\prime} t^{3}
$$

As $H$ is $(\rho, d)$-dense, we have

$$
e_{H}(\cup X) \geqslant d\left(\begin{array}{c}
\sum_{i \in X}\left|V_{i}\right| \\
3
\end{array}\right)-\rho n^{3} \geqslant d\left(\begin{array}{c}
|X| \\
3
\end{array}\right)(n / t)^{3}-\rho n^{3} .
$$

Indeed, the term $\left(\begin{array}{c}|X| \\ 3\end{array}\right)(n / t)^{3}$ accounts only for edges crossing with respect to $X$, while $\left(\underset{3}{\sum_{i \in X}\left|V_{i}\right|}\right)$ accounts also for triples inside clusters captured by $X$. By (5.2) we have $t+t^{2} \leqslant 2 t^{2} \leqslant \rho^{\prime} t^{3} / 4$, and by (5.3) we have $\rho \leqslant \rho^{\prime} / 4$. We may now write

$$
e_{R_{d^{\prime}}}(X) \geqslant \frac{d}{2}\left(\begin{array}{c}
|X| \\
3
\end{array}\right)-\left(\rho+\rho^{\prime} / 4+\varepsilon_{\text {reg }}+d^{\prime}\right) t^{3} \geqslant \frac{d}{2}\left(\begin{array}{c}
|X| \\
3
\end{array}\right)-\rho^{\prime} t^{3},
$$

and the claim follows.

In view of Claim 6 and the choice of $\rho^{\prime}$, it follows by Lemma 5.3 that $R_{d^{\prime}}$ admits a matching $M$ missing at most $\max \{2, \zeta t / 12\}$ vertices of $R_{d^{\prime}}$. For each edge $\left(V_{i}, V_{j}, V_{k}\right)$ of $M$, apply Lemma 5.2 to $H\left[V_{i}, V_{j}, V_{k}\right]$ to obtain a system of vertex-disjoint paths as described in Lemma 5.2. Let $\mathcal{P}$ denote the system of paths thus generated in $H$ over all edges of $M$. In each $H\left[V_{i}, V_{j}, V_{k}\right]$ corresponding to an edge $\left(V_{i}, V_{j}, V_{k}\right)$ of $M$, at most $3 /\left(\varepsilon_{\text {reg }}\left(d^{\prime}-\varepsilon_{\text {reg }}\right)\right)$ paths are packed. As $|M| \leqslant T_{5.1}\left(\varepsilon_{\text {reg }}, t_{\text {reg }}\right) / 3$, at most $\ell_{1.5}$ paths are thus packed.

It remains to argue that the members of $\mathcal{P}$ cover all but at most $\zeta n$ vertices of $H$. In each $H\left[V_{i}, V_{j}, V_{k}\right]$ corresponding to an edge $\left(V_{i}, V_{j}, V_{k}\right)$ of $M$, at most $2 \varepsilon_{\text {reg }} \cdot 6 n / t$ vertices of $H\left[V_{j}, V_{j}, V_{k}\right]$ are missed. As $|M| \leqslant t / 3$, at most $12 \varepsilon_{\text {reg }} n$ vertices of $H$ are missed this way. From the clusters not covered by $M$, at $\operatorname{most} \max \{2, \zeta t / 12\} \cdot 2 n / t$ vertices of $H$ are missed. Overall, at most $\left(12 \varepsilon_{\text {reg }}+\max \{4 / t, \zeta / 2\}\right) n$ vertices of $H$ are missed. Owing to (5.2), $12 \varepsilon_{\text {reg }} \leqslant \zeta / 2$ and $t \geqslant t_{\text {reg }} \geqslant 8 / \zeta$ (so that $4 / t \leqslant \zeta / 2$ ); consequently $12 \varepsilon_{\text {reg }}+\max \{12 / t, \zeta / 2\} \leqslant \zeta$ as required. 


\subsection{Path-covers in 3-set-dense 3-graphs}

In this section we provide a significantly shorter proof for Lemma 1.5, under the strengthened assumption that the host 3 -graph is $\therefore$-dense and not merely 1 -set-dense. The main ingredient, so to speak, of the argument for constructing path-covers in $\bullet-$-dense graphs is as follows.

Lemma 5.4. ([36, Claim 4.1]). Let $c>0$. Then every 3-partite 3-graph $H$ having at most $m$ vertices in each partition set and satisfying $e(H) \geqslant \mathrm{cm}^{3}$ contains a path on at least $\mathrm{cm}$ vertices.

Proof of Lemma 1.5 for $\bullet$-dense 3-graphs. Given $d:=d_{1.5}$ and $\zeta:=\zeta_{1.5}$, set $\rho_{1.5}:=d \zeta^{3} / 2 \cdot 3^{3}$, and set $\ell_{1.5}:=\left\lceil 1 / \rho_{1.5}\right\rceil$. Let $0<\rho<\rho_{1.5}$ be fixed, let $n$ be sufficiently large and let $H$ be a $(\rho, d)$ dense $n$-vertex 3-graph.

We define a sequence of subgraphs $H_{0}:=H \supseteq H_{1} \supseteq H_{2} \cdots$ as follows. Let $H_{i}$, for some $i \geqslant 0$, be given. If $n_{i}:=\left|V\left(H_{i}\right)\right|<\zeta n$, then set $H_{i+1}$ to be the empty graph. Otherwise note that $e_{H_{i}}\left(V_{1}, V_{2}, V_{3}\right)=e_{H}\left(V_{1}, V_{2}, V_{3}\right)$ whenever $V_{1}, V_{2}, V_{3} \subseteq V\left(H_{i}\right)$. Choose an arbitrary equipartition $V\left(H_{i}\right)=U_{1} \dot{\cup} U_{2} \dot{U} U_{3}$ such that $\left|U_{1}\right| \leqslant\left|U_{2}\right| \leqslant\left|U_{3}\right| \leqslant\left|U_{1}\right|+1$. Then $\left|U_{i}\right| \in\left\{n_{i} / 3, n_{i} / 3+1\right\}$, for every $i \in$ [3]. The 3-partite subgraph of $H_{i}$ induced by the edges of $H_{i} \operatorname{crossing} U_{1}, U_{2}$ and $U_{3}$ has $e_{H_{i}}\left(U_{1}, U_{2}, U_{3}\right)$ edges. For the latter quantity we observe that

$$
e_{H_{i}}\left(U_{1}, U_{2}, U_{3}\right)=e_{H}\left(U_{1}, U_{2}, U_{3}\right) \geqslant d\left|U_{1}\right|\left|U_{2}\right|\left|U_{3}\right|-\rho n^{3} \geqslant\left(\frac{d \zeta^{3}}{3^{3}}-\rho\right) n^{3} \geqslant \frac{d \zeta^{3}}{2 \cdot 3^{3}} n^{3} .
$$

As $\left|U_{j}\right| \leqslant 2 n / 3$ holds for every $j \in$ [3], it follows by Lemma 5.4 that $H_{i}$ contains a path $P_{i}$ of length at least $\left(d \zeta^{3} / 3^{4}\right) n$. Set $H_{i+1}:=H_{i}-V\left(P_{i}\right)$.

In the above sequence, all graphs $H_{i}$ with $i>\ell_{1.5}$ are empty. Hence at most $\ell_{1.5}$ paths are defined throughout the above process; these paths form the required path-cover of $H$.

\section{Proofs of the main results}

Let $H$ be a 3-graph, let $x, y \in V(H)$ and let $H^{\prime} \subseteq H$ with $\{x, y\} \nsubseteq V\left(H^{\prime}\right)$ possible. Define

$$
\operatorname{deg}_{H^{\prime}}(x, y):=\left|N_{H}(x, y) \cap V\left(H^{\prime}\right)\right| \text {. }
$$

\subsection{Proof of Theorem 1.2: 2-degree setting}

Given $d>0$ and $\alpha>0$, set

$$
0<\beta<\min \{d, \alpha\}, \quad \kappa:=\kappa_{1.3}(d, \alpha, \beta) \leqslant \beta / 2, \quad \mu:=\mu_{1.3}(d, \alpha, \beta), \quad v \ll \mu, \quad \zeta:=\mu-2 v .
$$

The inequality $\kappa \leqslant \beta / 2$ is supported by Lemma 1.3 . Set

$$
0<\rho<\min \left\{\rho_{1.3}(d, \alpha, \beta), 2^{-1}(1-\kappa-v)^{3} \cdot \rho_{1.5}(d, \zeta), \rho_{1.1}(d, \beta / 8) \cdot(v(1-\kappa) / 4)^{3}\right\} .
$$

Let $n$ be sufficiently large and let $H$ be an $n$-vertex $(\rho, d)$-dense 3-graph satisfying $\delta_{2}(H) \geqslant$ $\alpha(n-2)$. As $\rho<\rho_{1.3}(d, \alpha, \beta), H$ admits a $(\beta, \mu, \kappa)$-absorbing-path $A$, by Lemma 1.3. Define $H^{\prime}:=$ $H-V(A)$ (i.e. $H^{\prime}$ is attained from $H$ by removing the vertices of $A$ and all edges incident to $V(A)$ from $H)$.

We prepare for an application of Lemma 1.6. For each pair of vertices $\{x, y\} \in\left(\begin{array}{c}V(H) \\ 2\end{array}\right)$, set $U_{\{x, y\}}:=N_{H}(x, y) \cap V\left(H^{\prime}\right)$. Then

$$
\begin{aligned}
\operatorname{deg}_{H^{\prime}}(x, y) & =\left|U_{\{x, y\}}\right| \geqslant \alpha(n-2)-|V(A)| \geqslant \alpha(n-2)-\kappa n \\
& \geqslant(\alpha-\kappa) n-2 \alpha \stackrel{\alpha>\beta}{\geqslant}(\beta-\kappa) n \stackrel{(6.1)}{\geqslant} \beta n / 2
\end{aligned}
$$


for every $\{x, y\} \in\left(\begin{array}{c}V(H) \\ 2\end{array}\right)$. Lemma 1.6, applied with $v_{1.6}=v$, then asserts that there exists a set $R \subseteq$ $V\left(H^{\prime}\right)$ satisfying

$$
\left|N_{H}(x, y) \cap R\right| \geqslant\left(\beta / 2-2 n^{-1 / 3}\right)|R| \geqslant \beta|R| / 4
$$

for every $\{x, y\} \in\left(\begin{array}{c}V(H) \\ 2\end{array}\right)$. Consequently, $\delta_{2}(H[R]) \geqslant \beta|R| / 4$. Moreover, the set $R$ also satisfies $|R|=v n^{\prime} \pm v\left(n^{\prime}\right)^{2 / 3}$, where $n^{\prime}:=\left|V\left(H^{\prime}\right)\right| \geqslant(1-\kappa) n$. We may then write

$$
\frac{\nu(1-\kappa)}{2} n \leqslant|R| \leqslant 2 v n \text {. }
$$

Set $H^{\prime \prime}:=H^{\prime}-R$. Then

$$
n^{\prime \prime}:=\left|V\left(H^{\prime \prime}\right)\right| \geqslant n-|V(A)|-|R| \stackrel{(6.4)}{\geqslant} n-\kappa n-2 v n=(1-\kappa-2 v) n .
$$

Claim 7. $H^{\prime \prime}$ is $\left(\xi_{1}, d\right) \ldots$-dense for some $\xi_{1}<\rho_{1.5}(d, \zeta)$.

Proof. Fix $\vec{G}_{1}, \vec{G}_{2} \subseteq V\left(H^{\prime \prime}\right) \times V\left(H^{\prime \prime}\right)$ and note that as $H^{\prime \prime}$ is an induced subgraph of $H$, then

$$
\begin{aligned}
e_{H^{\prime \prime}}\left(\vec{G}_{1}, \vec{G}_{2}\right) & \geqslant d\left|\mathcal{P}_{2}\left(\vec{G}_{1}, \vec{G}_{2}\right)\right|-\rho n^{3} \\
& \stackrel{(6.2)}{\geqslant} d\left|\mathcal{P}_{2}\left(\vec{G}_{1}, \vec{G}_{2}\right)\right|-2^{-1} \cdot \rho_{1.5}(d, \zeta)(1-\kappa-2 v)^{3} n^{3} \\
& \stackrel{(6.5)}{\geqslant} d\left|\mathcal{P}_{2}\left(\vec{G}_{1}, \vec{G}_{2}\right)\right|-2^{-1} \cdot \rho_{1.5}(d, \zeta)\left(n^{\prime \prime}\right)^{3} .
\end{aligned}
$$

As $\therefore$-denseness implies 1-set-denseness, it follows that $H^{\prime \prime}$ is $\left(\xi_{1}, d\right)$-dense for some $\xi_{1}<$ $\rho_{1.5}(d, \zeta)$. Lemma 1.5 then asserts that $H^{\prime \prime}$ admits a collection $\mathcal{P}^{\prime}:=\left\{P_{1}, \ldots, P_{h-1}\right\}, h-1 \leqslant$ $\ell_{1.5}(d, \zeta)$, of vertex-disjoint paths covering all but at most $\zeta n^{\prime \prime} \leqslant \zeta n$ vertices of $H^{\prime \prime}$ and thus of $H$ as well. Write $P_{h}:=A$ and set $\mathcal{P}:=\mathcal{P}^{\prime} \cup\left\{P_{h}\right\}$.

In what follows we use the set $R$ in order to concatenate the members of $\mathcal{P}$ into a (tight) cycle. This entails $h$ applications of the connecting lemma suited to this setting, namely Lemma 1.1. We proceed in two steps. First, a path of the form

$$
L:=P_{1} \circ K_{1} \circ P_{2} \circ K_{2} \circ \cdots \circ K_{h-1} \circ P_{h}
$$

is constructed, where each $K_{i}$ is a 10-path disjoint of all other 10-paths involved in the construction. Second, the remaining 'free' end-pair of $P_{h}$ is connected using an additional 10-path with the remaining 'free' end-pair of $P_{1}$. The resulting cycle we denote by $C$. We now make this precise.

The construction of $L$ is done inductively. Set $L_{1}:=P_{1}$. Assuming

$$
L_{i}:=P_{1} \circ K_{2} \cdots \circ K_{i-1} \circ P_{i}
$$

has been constructed for some $i \in[h-1]$, we define $L_{i+1}$ as follows. Let $\{a, b\}$ be the free end-pair of $P_{i}$ and let $\{c, d\}$ be one of the end-pairs of $P_{i+1}$. Set

$$
R_{i}:=\left(R \backslash V\left(L_{i}\right)\right) \cup\{a, b, c, d\} .
$$

Observing that $\left|V\left(L_{i}\right) \cap R\right| \leqslant 10 h$, we may write that

$$
\left|R_{i}\right| \geqslant|R|-10 h \stackrel{(6.4)}{\geqslant} v(1-\kappa) n / 2-10 h \geqslant v(1-\kappa) n / 4
$$

for sufficiently large $n$. Owing to $\rho<\rho_{1.1}(d, \beta / 8) \cdot(v(1-\kappa) / 4)^{3}$, by $(6.2)$, an argument identical to that seen in Claim 7 establishes that $H\left[R_{i}\right]$ is $\left(\xi_{2}, d\right) \therefore$-dense for some $\xi_{2}<\rho_{1.1}(d, \beta / 4)$. Moreover,

$$
\delta_{2}\left(H\left[R_{i}\right]\right) \stackrel{(6.3)}{\geqslant} \beta|R| / 4-10 h \geqslant \beta|R| / 8 \geqslant \beta\left|R_{i}\right| / 8
$$


whenever $n$ is sufficiently large. The path $K_{i}$ connecting $\{a, b\}$ and $\{c, d\}$ in $H\left[R_{i}\right]$ then exists, by Lemma 1.1 applied with $d_{1.1}=d$ and $\alpha_{1.1}=\beta / 8$. This completes the construction of $L$. Completing $L$ into the aforementioned cycle $C$ is done using the exact same argument provided for $K_{i}$.

With respect to the original 3-graph $H$, the cycle $C$ covers all vertices of the latter but (a subset of) those found in $V\left(H^{\prime \prime}\right) \backslash V(\mathcal{P})$ and those vertices of $R$ not used throughout the construction of $C$. The number of uncovered vertices is then at most

$$
\left|V\left(H^{\prime \prime}\right) \backslash V(\mathcal{P})\right|+|R| \leqslant \zeta n+2 v n \stackrel{(6.1)}{=}(\mu-2 v) n+2 v n=\mu n .
$$

The path $A$, present in $C$, can then absorb all uncovered vertices, rendering a tight Hamilton cycle in $H$. This completes the proof of Theorem 1.2.

\subsection{Proof of Theorem 1.3: 1-degree setting}

Given $\alpha>0$ and $d>0$ satisfying $\alpha+d>1$, set

$$
\eta \ll \min \left\{g(\alpha, d), a+d-1, \frac{\alpha+d-1}{3(d+1)+2}\right\} .
$$

Recalling the definition of $g(\cdot)$ from (1.3), note that we have just imposed $\eta \ll \alpha$, $d$ as well. With $\eta$ set, define

$$
\kappa:=\kappa_{1.4}(d, \alpha, \eta) \ll \eta, \quad \mu:=\mu_{1.4}(d, \alpha, \eta), \quad v \ll \mu, \quad \zeta:=\mu-2 v,
$$

where the inequality $\kappa \ll \eta$ is supported by Lemma 1.4. As $\kappa \ll \eta \ll \alpha+d-1$, we may insist on $\alpha-\kappa+d-1>0$. Fix an auxiliary constant $0<\gamma \ll \eta$ such that

$$
\alpha-\kappa-\gamma+d-1>0 \text {. }
$$

Define an additional auxiliary constant $0<\gamma^{\prime} \ll \eta$ such that

$$
\eta+\kappa+\gamma^{\prime} \ll g(\alpha-\kappa-\gamma, d) \stackrel{(1.3)}{=} \min \left\{\alpha-\kappa-\gamma, d, \frac{\alpha-\kappa-\gamma+d-1}{d+1}\right\} .
$$

To see that (6.11) is possible, we iterate over each of the constraints involved in it. For the first one, note that the requirement $\eta+\kappa+\gamma^{\prime} \ll \alpha-\kappa-\gamma$ can be rewritten to read $\eta+2 \kappa+\gamma+\gamma^{\prime} \ll \alpha$. Owing to being able to choose $\kappa, \gamma, \gamma^{\prime} \ll \eta$, this amounts to requiring $\eta \ll \alpha$; the latter is already imposed on $\eta$. In a similar manner, the second constraint reading $\eta+\kappa+\gamma^{\prime} \ll d$ amounts to requiring $\eta \ll d$, which is also already imposed on $\eta$. Finally, for the third constraint appearing in (6.11), appeal again to the ability to choose $\kappa, \gamma, \gamma^{\prime} \ll \eta$, so that the third constraint amounts to requiring

$$
\eta \ll \frac{\alpha+d-1}{3(d+1)+2},
$$

which is also already imposed on $\eta$.

We conclude our selection of constants by setting

$$
\begin{array}{r}
0<\rho<\min \left\{\eta / 4, \rho_{1.4}(d, \alpha, \eta), 2^{-1} \eta(1-\kappa-2 v)^{3} \cdot \rho_{1.5}(d, \zeta)\right. \\
\left.\rho_{1.2}\left(d, \alpha-\kappa-\gamma, \eta+\kappa+\gamma^{\prime}\right) \cdot(\nu(1-\kappa) / 4)^{3}\right\} .
\end{array}
$$

Let $n$ be sufficiently large and let $H$ be an $n$-vertex $(\rho, d) \therefore$-dense 3-graph satisfying $\delta(H) \geqslant$ $\alpha\left(\begin{array}{c}n-1 \\ 2\end{array}\right)$. As $\rho<\rho_{1.4}(d, \alpha, \eta), H$ admits a $(d-\eta, \mu, \kappa)$-absorbing-path $A$, by Lemma 1.4. Define $H^{\prime}:=H-V(A)$. 
We prepare for an invocation of Lemma 1.6. Let $D_{d-\eta}(H)$ denote the set of pairs $\{x, y\} \in\left(\begin{array}{c}V(H) \\ 2\end{array}\right)$ satisfying $\operatorname{deg}_{H}(x, y) \geqslant(d-\eta) n$. Owing to $\eta \ll d$ and $\rho<\eta / 4$, by $(6.12)$, the set $D_{d-\eta}(H)$ has order of magnitude $\Omega\left(n^{2}\right)$, by (2.3). For each pair $\{x, y\} \in D_{d-\eta}(H)$, set

$$
U_{\{x, y\}}:=N_{H}(x, y) \cap V\left(H^{\prime}\right) .
$$

For each vertex $x \in V(H)$, let $L_{x}:=L_{x}(H)$ denote the link graph ${ }^{\mathrm{j}}$ of $x$ in $H$. Set $L_{x, H^{\prime}}$ to be the subgraph of $L_{x}$ induced by $V\left(H^{\prime}\right)$. For any pair $\{x, y\} \in D_{d-\eta}(H)$, we may write

$$
\operatorname{deg}_{H^{\prime}}(x, y)=\left|U_{\{x, y\}}\right| \geqslant \operatorname{deg}_{H}(x, y)-|V(A)| \geqslant(d-\eta-\kappa) n .
$$

For a vertex $x \in V(H)$, we may write

$$
\begin{aligned}
\operatorname{deg}_{H^{\prime}}(x) & =e\left(L_{x, H^{\prime}}\right) \\
& \geqslant e\left(L_{x}\right)-|V(A)| \cdot n \\
& \geqslant \alpha\left(\begin{array}{c}
n-1 \\
2
\end{array}\right)-\kappa n^{2} \\
& \geqslant(\alpha-\kappa) \frac{n^{2}}{2} \quad(\text { as seen in }(4.19)) \\
& \geqslant(\alpha-\kappa)\left(\begin{array}{c}
n^{\prime}-1 \\
2
\end{array}\right),
\end{aligned}
$$

where $n^{\prime}:=\left|V\left(H^{\prime}\right)\right|$.

Lemma 1.6, applied with $v_{1.6}=v$, then asserts that there exists a set $R \subseteq V\left(H^{\prime}\right)$ of size $|R|=$ $v n^{\prime} \pm v\left(n^{\prime}\right)^{2 / 3}$, and thus satisfying (6.4), such that

$$
\left|N_{H}(x, y) \cap R\right| \geqslant\left(d-\eta-\kappa-2 n^{-1 / 3}\right)|R|,
$$

whenever $\{x, y\} \in D_{d-\eta}(H)$, and such that, for every $x \in V(H)$,

$$
e\left(L_{x, H^{\prime}}[R]\right) \geqslant\left(\alpha-\kappa-3 n^{-1 / 3}\right)\left(\begin{array}{c}
|R| \\
2
\end{array}\right) .
$$

In particular,

$$
\delta(H[R]) \geqslant\left(\alpha-\kappa-3 n^{-1 / 3}\right)\left(\begin{array}{c}
|R| \\
2
\end{array}\right) .
$$

Define $H^{\prime \prime}:=H^{\prime}-R$. Then $n^{\prime \prime}:=\left|V\left(H^{\prime \prime}\right)\right|$ satisfies (6.5). This estimate, coupled with

$$
\rho \stackrel{(6.12)}{<} 2^{-1} \eta(1-\kappa-2 v)^{3} \cdot \rho_{1.5}(d, \zeta),
$$

and put through the argument seen in Claim 7, implies that

$$
H^{\prime \prime} \text { is }\left(\xi_{1}, d\right) \therefore \text {-dense for some } \xi_{1}<2^{-1} \eta \cdot \rho_{1.5}(d, \zeta) \text {. }
$$

Recall the definition put forth in Section 2 of the spanning subgraph $H_{d-\eta}^{\prime \prime} \subseteq H^{\prime \prime}$. In the following claim we essentially appeal to (2.4) asserting that $H_{d-\eta}^{\prime \prime}$ is $\therefore$-dense. Here, however, we require much less.

Claim 8. $H_{d-\eta}^{\prime \prime}$ is $\left(\xi_{2}, d\right)$-dense, for some $\xi_{2}<\rho_{1.5}(d, \zeta)$

Proof. Fix $X \subseteq V\left(H_{d-\eta}^{\prime \prime}\right)$. Then

$$
e_{H_{d-\eta}^{\prime \prime}}(X) \geqslant e_{H^{\prime \prime}}(X)-\left|B_{d-\eta}\left(H^{\prime \prime}\right)\right| \cdot n^{\prime \prime} .
$$

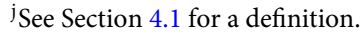


Writing $\eta=d-(d-\eta)$ and appealing to both (2.3) and (6.15), we may write

$$
\begin{aligned}
e_{H_{d-\eta}^{\prime \prime}}(X) & \geqslant d\left(\begin{array}{c}
|X| \\
3
\end{array}\right)-\frac{\rho_{1.5}(d, \zeta) \eta}{2}\left(n^{\prime \prime}\right)^{3}-\frac{\rho_{1.5}(d, \zeta) \cdot(d-(d-\eta))}{2 \cdot(d-(d-\eta))}\left(n^{\prime \prime}\right)^{3} \\
& \geqslant \leqslant 1\left(\begin{array}{c}
|X| \\
3
\end{array}\right)-\rho_{1.5}(d, \zeta)\left(n^{\prime \prime}\right)^{3} .
\end{aligned}
$$

As $H_{d-\eta}^{\prime \prime}$ spans $H^{\prime \prime},\left|V\left(H_{d-\eta}^{\prime \prime}\right)\right|=n^{\prime \prime}$ holds, and the claim follows.

Lemma 1.5 then asserts that $H_{d-\eta}^{\prime \prime}$ admits a collection $\mathcal{P}^{\prime}:=\left\{P_{1}, \ldots, P_{h-1}\right\}, h-1 \leqslant \ell_{1.5}(d, \zeta)$, of vertex-disjoint paths covering all but at most $\zeta n^{\prime \prime} \leqslant \zeta n$ vertices of $H^{\prime \prime}$ (recall that $H_{d-\eta}^{\prime \prime}$ spans $\left.H^{\prime \prime}\right)$ and thus of $H$ as well. Write $P_{h}:=A$ and set $\mathcal{P}:=\mathcal{P}^{\prime} \cup\left\{P_{h}\right\}$. By definition of $A$ and $H_{d-\eta}^{\prime \prime}$,

$$
\{x, y\} \in D_{d-\eta}(H) \text { whenever }\{x, y\} \text { is an end-pair of some path } P \in \mathcal{P} .
$$

As in the proof of Theorem 1.2, we seek to construct a path $L$ of the form (6.6) and then close the latter into a (tight) cycle $C$. With the approach for this construction here conceptually identical to that seen in the proof of Theorem 1.2, we focus on the differences. More specifically, we are to show that $h$ applications of the connecting lemma relevant to the setting at hand, namely Lemma 1.2, can be carried out to construct the cycle $C$. To that end, for $i \in[h-1]$, define $L_{i}$ and $R_{i}$ as in (6.7) and (6.8), respectively. The following series of claims verifies that $H\left[R_{i}\right]$ adheres to the premise of Lemma 1.2 so that the pairs $\{a, b\}$ and $\{c, d\}$ (per the definition of $R_{i}$ ) can be connected through $H\left[R_{i}\right]$.

Claim 9. $\delta\left(H\left[R_{i}\right]\right) \geqslant(\alpha-\kappa-\gamma)\left(\begin{array}{c}|R| \\ 2\end{array}\right)$.

Proof. Observe that

$$
\delta\left(H\left[R_{i}\right]\right) \stackrel{(6.14)}{\geqslant} \delta(H[R])-10 h \cdot n \geqslant\left(\alpha-\kappa-3 n^{-1 / 3}\right)\left(\begin{array}{c}
|R| \\
2
\end{array}\right)-10 h \cdot n,
$$

where the term $10 h \cdot n$ accounts for all pairs involving a vertex in $V\left(L_{i}\right) \cap R$. As $10 h \cdot n=O(n)$ (recall that $h-1 \leqslant \ell_{1.5}(d, \zeta)$ ) and $|R|=\Omega(n)$, by (6.4), the claim follows.

Next we consider the codegree of the pairs $\{a, b\}$ and $\{c, d\}$, per the definition of $R_{i}$.

Claim 10. $\operatorname{deg}_{H\left[R_{i}\right]}(a, b), \operatorname{deg}_{H\left[R_{i}\right]}(a, b) \geqslant\left(d-\eta-\kappa-\gamma^{\prime}\right)|R|$

Proof. By (6.16), the pairs $\{a, b\},\{c, d\}$ lie in $D_{d-\eta}(H)$. Consequently (6.13) holds for both these pairs. Then

$$
\operatorname{deg}_{H\left[R_{i}\right]}(a, b) \geqslant\left(d-\eta-\kappa-2 n^{-1 / 3}\right)|R|-10 h .
$$

As $10 h=O(1)$ and $|R|=\Omega(n)$, by (6.4), the claim follows for $\{a, b\}$. A similar argument holds for $\{c, d\}$.

Next we address the $\therefore$-denseness of $H\left[R_{i}\right]$.

Claim 11. $H\left[R_{i}\right]$ is $\left(\xi_{3}, d\right)_{\therefore}$-dense, for some $\xi_{3}<\rho_{1.2}\left(d, \alpha-\kappa-\gamma, \eta+\kappa+\gamma^{\prime}\right)$. 
Proof. The set $R_{i}$ satisfies (6.9). Then, owing to

$$
\rho<\rho_{1.2}\left(d, \alpha-\kappa-\gamma, \eta+\kappa+\gamma^{\prime}\right) \cdot(\nu(1-\kappa) / 4)^{3},
$$

an argument identical to that seen in Claim 7 establishes the claim.

Owing to (6.10) and (6.11), $\alpha-\kappa-\gamma+d-1>0$ and $\eta+\kappa+\gamma^{\prime}<g(\alpha-\kappa-\gamma, d)$ hold, respectively. These inequalities together with Claims 9,10 and 11 collectively assert that $H\left[R_{i}\right]$ and the pairs $\{a, b\}$ and $\{c, d\}$ satisfy the premise of Lemma 1.2 with $\alpha_{1.2}=\alpha-\kappa-\gamma, d_{1.2}=d$ and $\eta_{1.2}=\eta+\kappa+\gamma^{\prime}$. The pairs $\{a, b\}$ and $\{c, d\}$ can then indeed be connected through $H\left[R_{i}\right]$ as alleged.

This completes the definition of the cycle $C$ containing the absorbing-path $A$. To prove that $C$ can be extended to absorb all possibly uncovered vertices, use the argument seen for this in the proof of Theorem 1.2. This concludes our proof of Theorem 1.3.

\section{Acknowledgements}

We would like to thank Yury Person and Mathias Schacht for their helpful remarks on an earlier draft of the manuscript. We are greatly indebted to two anonymous referees for their meticulous dedication towards improving this manuscript, for making numerous suggestions, finding mistakes, and overall treating this manuscript as their own.

\section{References}

[1] Aigner-Horev, E., Conlon, D., Hàn, H., Person, Y. and Schacht, M. (2018) Quasirandomness in hypergraphs. Electron. J. Combin. 25 \#3.34.

[2] Araújo, P., Piga, S. and Schacht, M. (2019) Localised codegree conditions for tight Hamiltonian cycles in 3-uniform hypergraphs. Acta Math. Univ. Comenian. 88 389-394.

[3] Blakley, G. R. and Roy, P. (1965) A Hölder type inequality for symmetric matrices with nonnegative entries. Proc. Amer. Math. Soc. 16 1244-1245.

[4] Buß, E., Hàn, H. and Schacht, M. (2013) Minimum vertex degree conditions for loose Hamilton cycles in 3-uniform hypergraphs. J. Combin. Theory Ser. B 103 658-678.

[5] Chung, F. R. K., Graham, R. L. and Wilson, R. M. (1989) Quasi-random graphs. Combinatorica 9 345-362.

[6] Conlon, D., Hàn, H., Person, Y. and Schacht, M. (2012) Weak quasi-randomness for uniform hypergraphs. Random Struct. Algorithms 40 1-38.

[7] Cooley, O. and Mycroft, R. (2017) The minimum vertex degree for an almost-spanning tight cycle in a 3-uniform hypergraph. Discrete Math. 340 1172-1179.

[8] De Oliveira Bastos, J., Mota, G. O., Schacht, M., Schnitzer, J. and Schulenburg, F. (2017) Loose Hamiltonian cycles forced by large $(k-2)$-degree: approximate version. SIAM J. Discrete Math. 31 2328-2347.

[9] De Oliveira Bastos, J., Mota, G. O., Schacht, M., Schnitzer, J. and Schulenburg, F. (2018) Loose Hamiltonian cycles forced by large $(k-2)$-degree: sharp version. Contrib. Discrete Math. 13 88-100.

[10] Diestel, R. (2010) Graph Theory, fourth edition, Vol. 173 of Graduate Texts in Mathematics. Springer.

[11] Dirac, G. A. (1952) Some theorems on abstract graphs. Proc. London Math. Soc. (3) 2 69-81.

[12] Erdős, P. and Simonovits, M. (1984) Cube-supersaturated graphs and related problems. In Progress in Graph Theory (Waterloo, Ont., 1982) (J. A. Bondy and U. S. R. Murty, eds), pp. 203-218. Academic Press.

[13] Glebov, R., Person, Y. and Weps, W. (2012) On extremal hypergraphs for Hamiltonian cycles. European J. Combin. 33 544-555.

[14] Hàn, H. and Schacht, M. (2010) Dirac-type results for loose Hamilton cycles in uniform hypergraphs. J. Combin. Theory Ser. B $100332-346$.

[15] Han, J. and Zhao, Y. (2015) Minimum codegree threshold for Hamilton $\ell$-cycles in $k$-uniform hypergraphs. J. Combin. Theory Ser. A 132 194-223.

[16] Han, J. and Zhao, Y. (2015) Minimum vertex degree threshold for loose Hamilton cycles in 3-uniform hypergraphs. J. Combin. Theory Ser. B 114 70-96.

[17] Han, J. and Zhao, Y. (2016) Forbidding Hamilton cycles in uniform hypergraphs. J. Combin. Theory Ser. A 143 107-115.

[18] Janson, S., Łuczak, T. and Ruciński, A. (2000) Random Graphs, Wiley-Interscience Series in Discrete Mathematics and Optimization. Wiley-Interscience. 
[19] Katona, G. Y. and Kierstead, H. A. (1999) Hamiltonian chains in hypergraphs. J. Graph Theory 30 205-212.

[20] Keevash, P., Kühn, D., Mycroft, R. and Osthus, D. (2011) Loose Hamilton cycles in hypergraphs. Discrete Math. 311 544-559.

[21] Krivelevich, M. and Sudakov, B. (2006) Pseudo-random graphs. In More Sets, Graphs and Numbers, Vol. 15 of Bolyai Society Mathematical Studies, pp. 199-262. Springer.

[22] Kühn, D., Mycroft, R. and Osthus, D. (2010) Hamilton $\ell$-cycles in uniform hypergraphs. J. Combin. Theory Ser. A 117 910-927.

[23] Kühn, D. and Osthus, D. (2006) Loose Hamilton cycles in 3-uniform hypergraphs of high minimum degree. J. Combin. Theory Ser. B 96 767-821.

[24] Kühn, D. and Osthus, D. (2014) Hamilton cycles in graphs and hypergraphs: an extremal perspective. In Proceedings of the International Congress of Mathematicians 2014, Seoul, Korea, pp. 381-406.

[25] Lenz, J. and Mubayi, D. (2015) Eigenvalues and linear quasirandom hypergraphs. Forum Math. Sigma 3 e2, 26.

[26] Lenz, J. and Mubayi, D. (2015) The poset of hypergraph quasirandomness. Random Struct. Algorithms $46762-800$.

[27] Lenz, J., Mubayi, D. and Mycroft, R. (2016) Hamilton cycles in quasirandom hypergraphs. Random Struct. Algorithms 49 363-378.

[28] Reiher, C., Rödl, V. and Schacht, M. (2016) Embedding tetrahedra into quasirandom hypergraphs. J. Combin. Theory Ser. B 121 229-247.

[29] Reiher, C., Rödl, V. and Schacht, M. (2018) Some remarks on $\pi_{\wedge}$. In Connections in discrete mathematics, pp. $214-239$. Cambridge University Press.

[30] Reiher, C., Rödl, V., Ruciński, A., Schacht, M. and Szemerédi, E. (2019) Minimum vertex degree condition for tight Hamiltonian cycles in 3-uniform hypergraphs. Proc. Lond. Math. Soc. (3) 119 409-439.

[31] Reiher, C. and Schacht, M. Private communication.

[32] Rödl, V. and Ruciński, A. (2010) Dirac-type questions for hypergraphs: a survey (or more problems for Endre to solve). In An Irregular Mind, Vol. 21 of Bolyai Society Mathematical Studies, pp. 561-590. János Bolyai Mathematical Society.

[33] Rödl, V. and Ruciński, A. (2014) Families of triples with high minimum degree are Hamiltonian. Discuss. Math. Graph Theory 34 361-381.

[34] Rödl, V., Ruciński, A., Schacht, M. and Szemerédi, E. (2017) On the Hamiltonicity of triple systems with high minimum degree. Ann. Combin. 21 95-117.

[35] Rödl, V., Ruciński, A. and Szemerédi, E. (2006) A Dirac-type theorem for 3-uniform hypergraphs. Combin. Probab. Comput. 15 229-251.

[36] Rödl, V., Ruciński, A. and Szemerédi, E. (2008) An approximate Dirac-type theorem for k-uniform hypergraphs. Combinatorica 28 229-260.

[37] Rödl, V., Ruciński, A. and Szemerédi, E. (2009) Perfect matchings in large uniform hypergraphs with large minimum collective degree. J. Combin. Theory Ser. A 116 613-636.

[38] Rödl, V., Ruciński, A. and Szemerédi, E. (2011) Dirac-type conditions for Hamiltonian paths and cycles in 3-uniform hypergraphs. Adv. Math. 227 1225-1299.

[39] Sidorenko, A. F. (1986) Extremal problems in graph theory and functional-analytic inequalities. In Proceedings of the All-Union Seminar on Discrete Mathematics and its Applications (Moscow, 1984), pp. 99-105 (in Russian). Moskov. Gos. Univ., Mekh.-Mat. Fak.

[40] Szemerédi, E. (1978) Regular partitions of graphs. In Problèmes Combinatoires et Théorie des Graphes (Colloq. Internat. CNRS, Univ. Orsay, Orsay, 1976), Vol. 260 of Colloq. Internat. CNRS, pp. 399-401. CNRS.

[41] Thomason, A. (1987) Pseudorandom graphs. In Random Graphs '85 (Poznań, 1985), Vol. 144 of North-Holland Mathematics Studies, pp. 307-331. North-Holland.

[42] Thomason, A. (1987) Random graphs, strongly regular graphs and pseudorandom graphs. In Surveys in Combinatorics 1987 (New Cross, 1987), Vol. 123 of London Mathematical Society Lecture Note Series, pp. 173-195. Cambridge University Press.

[43] Towsner, H. (2017) $\sigma$-algebras for quasirandom hypergraphs. Random Struct. Algorithms 50 114-139.

[44] Zhao, Y. (2016) Recent advances on Dirac-type problems for hypergraphs. In Recent Trends in Combinatorics, Vol. 159 of IMA Volumes in Mathematics and its Applications, pp. 145-165. Springer.

Cite this article: Aigner-Horev E and Levy G (2021). Tight Hamilton cycles in cherry-quasirandom 3-uniform hypergraphs. Combinatorics, Probability and Computing 30, 412-443. https://doi.org/10.1017/S0963548320000486 